\title{
Meteorología y climatología de la vertiente del Pacífico colombiano
}

\author{
Hans Trojer
}

Rev. Acad. Colomb. Cienc. Ex. Fis. Nat. 1958, 10 (40): 199-219.

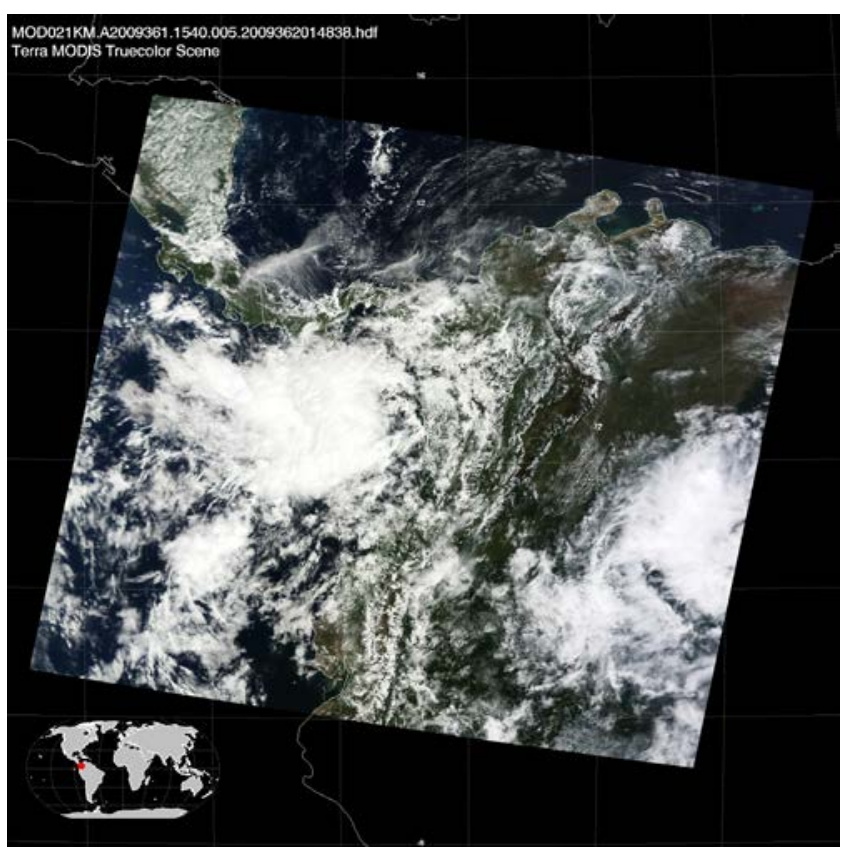

\section{Hans Trojer (1916-ND)}

Hans Trojer nació en 1916 en Klagenfurt, Austria. En 1939 se graduó como Doctor en Meteorología y Geofísica en la Universidad de Graz. Allí mismo trabajó como asistente científico entre 1938 y 1948. Posteriormente de 1940 a 1945 fue Meteorólogo de la Fuerza Aérea (Luftwaffe). Entre 1949 y 1951 trabajó como Geofísico del Servicio de Minería en la República Dominicana. Entre 1951 y 1965 trabajó como jefe de Agroclimatología de la Federación Nacional de Cafeteros de Colombia, en CENICAFE. Después de 1965 se desempeñó como Climatólogo y Asesor Principal de Enseñanza e Investigación en el Instituto Interamericano de Ciencias Agrícolas de la OEA, en Turrialba, Costa Rica. De sus trabajos en Colombia también se destacan: (1) "El tiempo reinante en Colombia, sus características y su desarrollo: Estudios básicos para una climatología dinámica de Colombia" (1954), y (2) "Fundamentos para una zonificación meteorológica y climatológica del trópico y especialmente de Colombia" (1959), ambos publicados por la Revista Cenicafé.
Buena parte del entendimiento que hoy tenemos sobre la variabilidad espacial y temporal de los procesos que definen el clima (largo plazo) y el tiempo atmosférico (corto plazo) en Colombia, se deben a H. Trojer. En particular, en este trabajo se discuten los principales mecanismos dinámicos y termodinámicos asociados con ambas escalas de tiempo, y es pionero en distinguir entre los fenómenos que dan lugar al clima, como la oscilación de la Zona de Convergencia Intertropical, y su influencia en el ciclo anual de la precipitación, de aquellos efectos locales causantes del tiempo atmosférico como la topografía y los sistemas locales de circulación (tierra-mar o valle-montaña).

La vertiente del Pacífico Colombiano es uno de los lugares más lluviosos del planeta, con precipitaciones promedias entre 8.000 y $13.000 \mathrm{~mm}$ por año. A partir de registros de diversas variables meteorológicas, este trabajo de Trojer discute la dinámica temporal y espacial de la meteorología y el clima de esta fascinante región de Colombia. Además, es pionero en temas como: (1) el ciclo diurno de la precipitación en Colombia, al evidenciar su alta variabilidad en las zonas de montaña, al estudiar su comportamiento en los distintos meses del año y su relación con la latitud, y al descubrir los mecanismos causantes del carácter bimodal de dicho ciclo diurno. (2) El ciclo anual de intensidad, frecuencia y duración de aguaceros. (3) El primer mapa de la distribución de la lluvia promedio anual sobre la región de estudio, al evidenciar una fuerte zonificación norte-centrosur. (4) La influencia entre la altura topográfica y las precipitaciones anuales. (5) Vincular dos variables que representan el balance de agua y el balance de energía, tales como la precipitación (P) y el brillo solar (B) con el fin de establecer un cociente que permitiera una zonificación climática, un tema de fundamental relevancia en la hidroecología actual.

Germán Poveda, Ph.D.

Miembro de Número 


\title{
METEOROLOGIA Y CLIMATOLOGIA DE LA VERTIENTE DEL PACIFICO COLOMBIANO
}

\author{
(UNA CONTRIBUCION AL ESTUDIO DEL "TIEMPO REINANTE EN COLOMBIA")
}

\author{
HANS TROJER \\ Jefe Sección de Meteorología, Centro Nal. \\ de Inv. de Café - Chinchiná
}

\begin{abstract}
El presente trabajo es una ampliación del presentado al simposium sobre ZONAS HUMEDAS TROPICALES reunido en Quibdó, bajo los auspicios de la UNESCO y el Gobierno Nacional de Colombia, durante el mes de marzo de 1958.

Aunque no puede hacerse una descripción completa, sin embargo se intenta captar los principales procesos dinámicos de la atmósfera y su relación con la topografía, que por medio de mediciones directas nunca se podría realizar, debido a la imposibilidad de aumentar las estaciones meteorológicas en una red tan densa como la que requeriría un país montañoso de tan variadas modificaciones locales.
\end{abstract}

\section{INTRODUCCION}

La observación meteorológica, muy escasa y discontinua, como ocurre generalmente en las zonas tropicales y especialmente en las húmedas, dificulta la interpretación de los datos correspondientes. Aunque algunos autores han hecho intentos de descripción del clima de la zona húmeda de la vertiente colombiana del Pacífico (13), dando las referencias más notorias, los métodos empleados no han logrado la generalización que se puede intentar por medio de una interpretación dinámica de las condiciones atmosféricas en relación con las características específicas de la topografía de esta región.

La deducción lógica según los conocimientos de la Meteorología General, y la comprobación de ciertos procesos fundamentales en el desarrollo del tiempo en el Trópico constituyen la base para la descripción dinámica del clima sobre las zonas de muy escasos datos meteorológicos. Naturalmente, hay que tomar en cuenta, también, las condiciones especiales debidas a la localización topográfica, como son las regiones costeras y los valles interiores o su extensión geográfica. En esta forma es posible llegar a conclusiones preliminares sobre la $\mathrm{Me}$ teorología y la Climatología tropical en general, y de las zonas húmedas más específicamente, si se adicionan para la interpretación algunas características comunes de tales zonas. Una comprobación semejante ha sido estudiada para otras regiones del territorio colombiano, como diferentes zonas cafeteras, la sabana de Bogotá, la cuenca de Medellín, y el valle del río Cauca (12) en los Departamentos de Cauca, Valle y Caldas, utilizando datos obtenidos por redes meteorológicas relativamente densas.

Esta interpretación, según la Climatología Dinámica desarrollada recientemente en varios centros de investigación climatológica, requiere mayores conocimientos de la meteorología y aventaja la acostumbrada por su descripción más completa y generalizable.

Como la observación de la atmósfera implica la medición separada de diferentes elementos, se ha acostumbrado la tendencia a enfocar también la interpretación climatológica según uno o varios de estos elementos. Esto ha sido motivado también por la necesidad de aplicación inmediata de los conocimientos para determinados fines prácticos, como por ejemplo la preferencia por la pluviosidad. Pero, no son sólo los pocos elementos de común interés los que definen la situación meteorológica de una región, ya que cada uno de ellos caracteriza únicamente en grado limitado el tiempo momentáneo o el tiempo predominante durante cierto lapso. Por esto, la climatología con su aplicación múltiple en las diversas actividades de la vida humana, no puede limitarse al estudio de uno u otro elemento perdiendo, así, la vista global.

En todo caso, de los fines de la interpretación y de su aplicación depende la inconveniencia o la insuficiencia de utilizar un solo elemento meteorológico. Tomando como ejemplo el caso del empleo de los datos de lluvia, las sumas anuales o mensuales no expresan suficientemente la distribución de los diversos sumandos, ni su magnitud, mostrándose por sumas iguales muy diferentes causas y efectividad de las mismas. En cambio, la interpretación por medio de los sistemas dinámicos permite determinar características importantes adicionales debido al reconocimiento de las causas físicas inmediatas, a la descripción del desarrollo y de la interacción de los factores más importantes; así como por la sucesión de un estado al otro de acuerdo con el cambio resultante de esta transformación por los mismos procesos dinámicos. Estudiando en esta forma los datos de cualquier región, el reconocimiento de la situación del tiempo depende de la separación de los principales factores actuantes, eligiendo adecuadamente los elementos más representativos. En consecuencia, pocas observaciones son necesarias, cuando ellas mismas permiten definir las características esenciales del tiempo momentáneo o del tiempo predominante durante épocas determinadas (secas y lluviosas), precisando la situación del tiempo reinante en toda la región. Por esto, cada observación es una muestra para la combinación específica entre la topografía y las condiciones atmosféricas, lo cual produce bajo las mismas relaciones manifestaciones semejantes del tiempo reinante.

\section{METEOROLOGIA DE LAS ZONAS HUMEDAS DEL TROPICO}

En consecuencia, con la introducción anterior, el tiempo reinante sobre una región que abarca zonas extensas puede ser bien identificado por los datos meteorológicos de una estación, cuando se toman en cuenta las caracte- 
rísticas topográficas del lugar y las modificaciones del tiempo debidas a tales circunstancias. Estos fenómenos locales, como todos los procesos de la atmósfera, obedecen a las leyes de la física y permiten por analogía la ampliación a zonas más vastas teniendo en cuenta estas mismas leyes. De ahí que los datos de las pocas estaciones de que se dispone hayan permitido hacer un estudio preliminar, pues las condiciones que reinan en las zonas húmedas del trópico, no son más que casos especiales de los fenómenos conocidos por la meteorología general en todas partes. Sería difícil determinar por medición directa, la gran variación que presentan los fenómenos meteorológicos en el trópico montañoso, ya que esto implicaría una red tan densa que ningún país del mundo podría sostenerla (5).

La zona húmeda del trópico se caracteriza, primordialmente, por el alto contenido de humedad del aire en el estado inicial de los procesos dinámicos ( 1 metro cúbico de aire en estado de saturación y a $30^{\circ} \mathrm{C}$, contiene 32 gramos de vapor de agua; a $20^{\circ} \mathrm{C}, 18$ gramos y a $10^{\circ} \mathrm{C}, 9$ gramos); así se explica la alta cantidad de agua en las nubes tropicales, y la consecuente abundancia de lluvia en estas zonas. Tales condiciones se presentan pronunciadamente cuando masas de aire ecuatorial y marí- timo fluyen hacia la línea de convergencia intertropical que se encuentra en las latitudes superiores. Además, la abundante pluviosidad mantiene la humedad relativa a un alto nivel muy próximo al estado de saturación y, en: estas condiciones, un reducido enfriamiento produce la condensación de estas enormes cantidades de vapor acuoso. El enfriamiento ocurre bien al ascender el aire, bien por la irradiación nocturna de la tierra, o por la mezcla o contacto inmediato con aire frío.

En la atmósfera tridimensional hay movimientos verticales y horizontales. El movimiento vertical (ascenso) depende de las condiciones topográficas y también del grado de la inestabilidad en la estructura vertical de las masas de aire actuantes (en analogía con la mecánica se conocen las estructuras estable, indiferente, e inestable). Un volumen de aire que empieza a ascender debido al levantamiento por la topografía o al calentamiento local (convección), sigue el proceso cuando, por la disminución matemática de la temperatura según las leyes termodinámicas, este volumen del aire está más cálido que los alrededores; es decir, la distribución vertical real de la temperatura corresponde a la situación inestable (Gráf. 1). Durante el tiempo anticiclonal (buen tiempo) se reducen las condiciones favorables para el movimiento asGRAFICO $\mathrm{N}^{2}$ ।

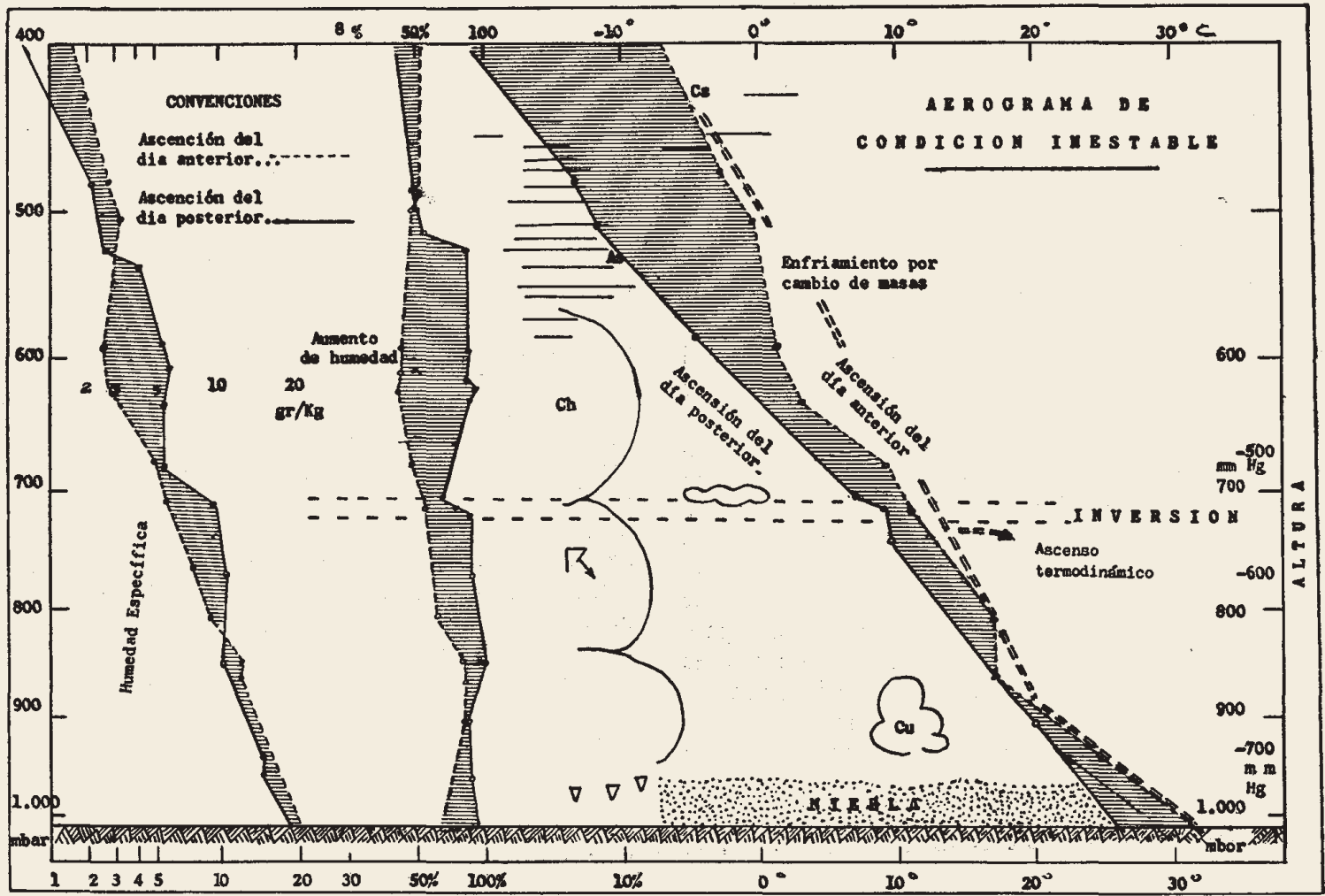

cendente (situación más estable), debido a las fuertes inversiones térmicas producidas por el efecto de subsidencia al enjutarse la zona de alta presión cuando el aire inferior se dispersa. En contraste, durante el tiempo ciclonal (mal tiempo) la afluencia de aires marítimos frescos.y el diferente calentamiento local, aumentan la estructura inestable y favorecen las condiciones para fuertes movimientos ascendentes (más de 15 metros por segundo) y con ellos la formación de nubes del tipo de cumulonimbos.

El movimiento horizontal, dependiente de la distribución bárica (centros de alta y baja presión atmosférica en los diferentes niveles altimétricos), determina algunas características típicas, según su origen, y la estructura de las masas de aire. Las condiciones de estructura se conservan principalmente durante su translación. La forma aerodinámica de las corrientes (convergencia o divergencia), influye además sobre el carácter del tiempo reinante; la convergencia produce movimientos verticales ascendentes con aumento de la nubosidad y por el contrario, la divergencia hace descender el aire y por consiguiente disuelve las nubes. El carácter de la topografía también se refleja sobre la corriente predominante en las manifestaciones del tiempo en la parte montañosa del 


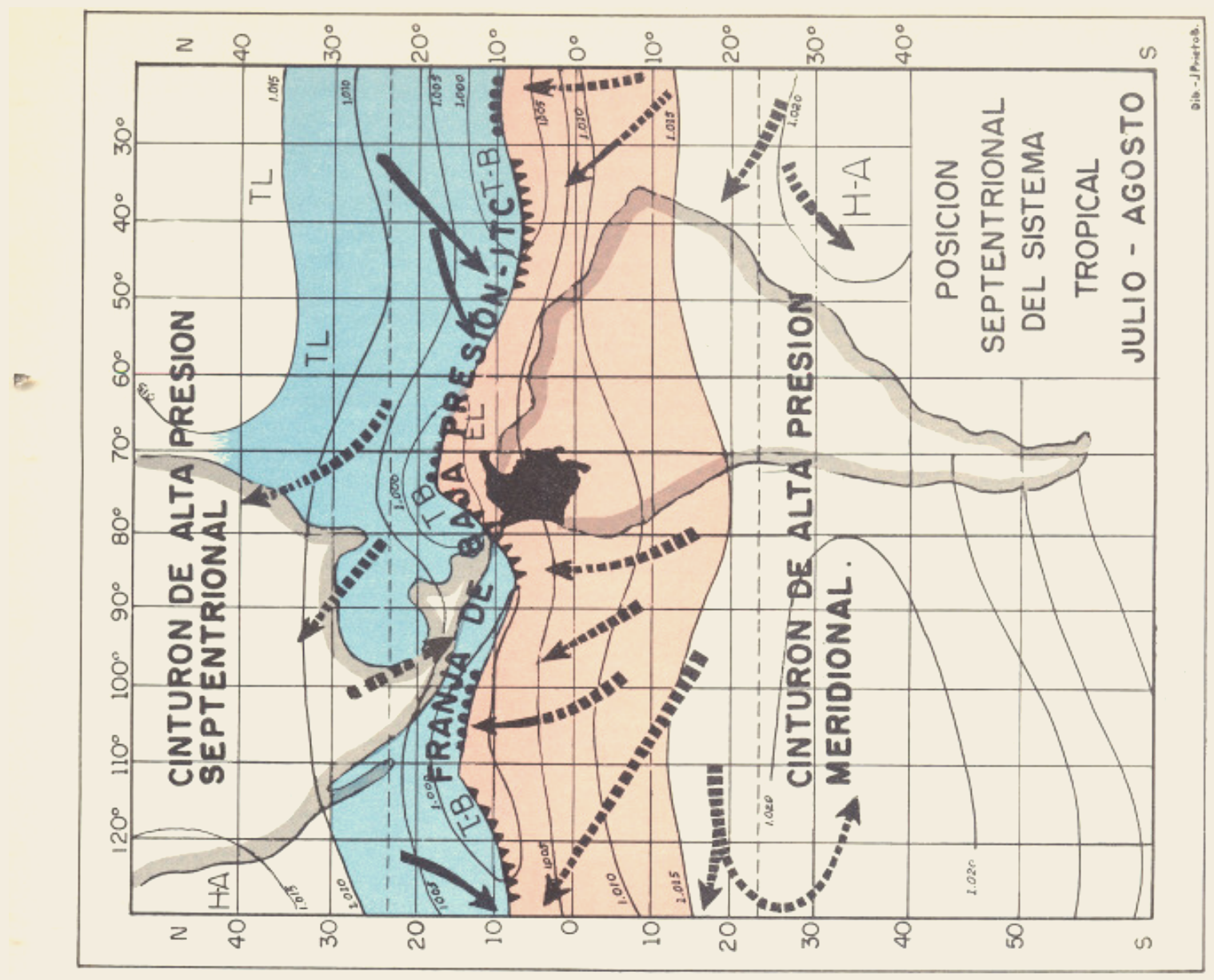

$\forall$
0
$z$
0
$u$
$\frac{u}{4}$
$\frac{\alpha}{0}$
0

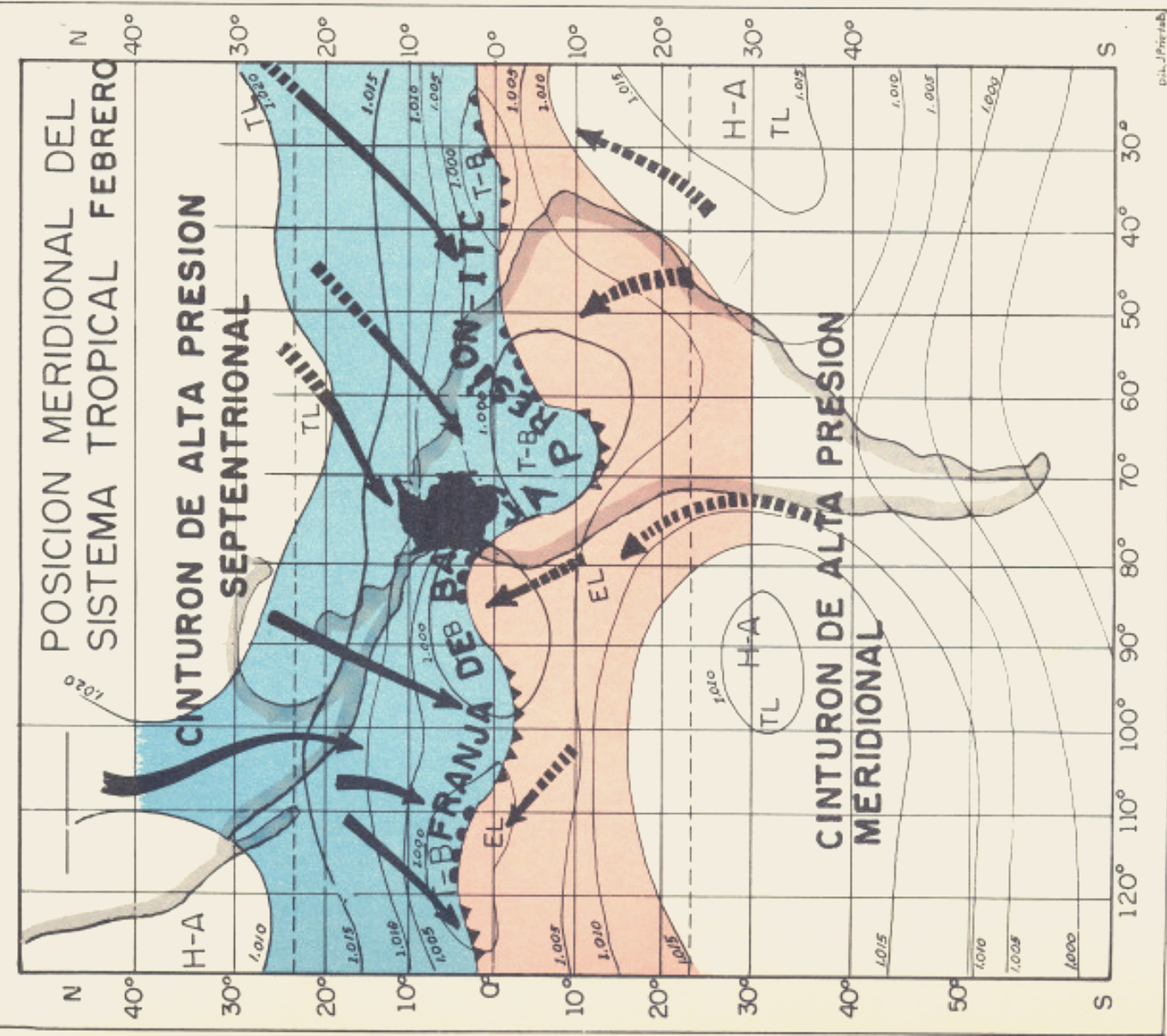

$m$
0
$z$
0
$\frac{U}{4}$
$\frac{1}{0}$
0 
trópico por los efectos de Estancamiento y Foehn. Estancamiento se correlaciona con levantamiento y por consiguiente con enfriamiento de las masas de aire ascendente y formación de nubes; mientras que Foehn se denomina el efecto del aire descendente en la parte de barlovento de la montaña donde se despeja el cielo.

Además de los fenómenos de las corrientes generales, en los países montañosos se presentan circulaciones verticales de carácter local (Gráf. 2) causadas por las diferencias térmicas entre valle y montaña, como también entre mar y costa, las cuales poseen su ciclo diario. Su grado de desarrollo depende de la intensidad de la radiación (insolación e irradiación nocturna) y de su consecuente transformación calórica, la cual, a su vez, cambia con la nubosidad formada y con la estructura del aire. La nubosidad densa y las precipitaciones pueden invertir la situación térmica diferencial de tal modo que las circulaciones también se invierten en horas fuera del ciclo normal (posición solar), como se observa en días

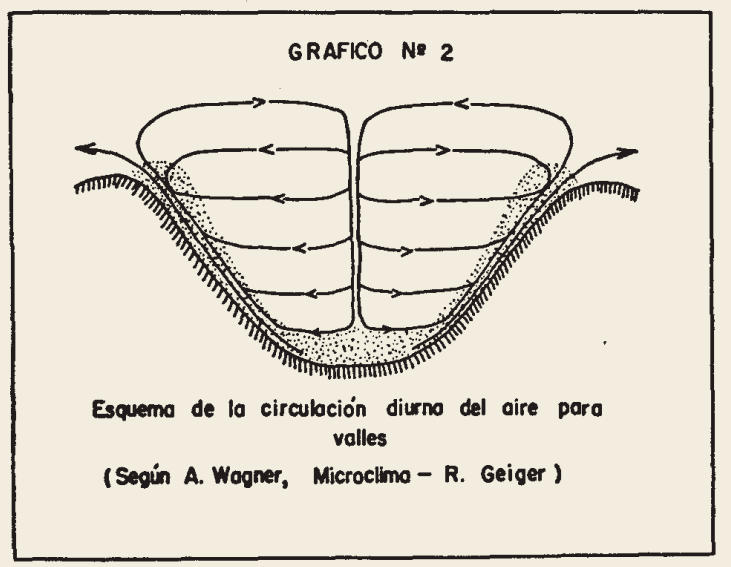

con varios cambios repentinos de nubosidad. Por condiciones especiales de topografía, las circulaciones se desarrollan con especial intensidad sobre determinados núcleos de las cimas de las montañas como lo demuestran la nubosidad local durante buen tiempo y la gran variación en la distribución pluvial. Estas diferencias locales son aún más pronunciadas en la zona chocoana que en el resto del país, debido a la gran inestabilidad de las masas de aire marítimo y a la gran disposición de la atmósfera para el desarrollo de movimientos verticales.

De acuerdo con lo anterior, el tiempo reinante depende, en general, de los siguientes factores (3):

A.-Macrotiempo, la situación momentánea de los centros de presión atmosférica que forman el sistema intertropical de circulación y que determinan la dirección de la translación horizontal y la estructura de las masas de aire, como también los efectos hidrodinámicos de convergencia y divergencia.

A. a.-Fenómenos locales producidos por la topografía, y por la corriente general según el macrotiempo (estancamiento y foehn).

B.-Sistemas locales de circulación recíproca (tierra mar o valle - montaña).

La combinación en cualquier grado de los factores anotados, determina el carácter del tiempo en cualquier momento y para cualquier lugar. Los mismos conceptos aplicados a los tiempos reinantes predominantes durante determinados períodos (tiempos lluviosos o de "invierno", tiempos secos "verano") son la base para la climatología de interpretación más amplia.
CLIMATOLOGIA BASICA DESCRIPTIVA DE LA ZONA HUMEDA DEL PACIFICO EN COLOMBIA

Entendiendo como clima el estado medio de la atmósfera sobre un lugar determinado, este está representado también, de acuerdo con el concepto de la climatología dinámica (6), por el tiempo reinante predominante sobre cada región durante épocas bien diferenciadas como las secas o las lluviosas. Tal distribución depende, en primer lugar, de la posición del sistema de circulación intertropical con su translación cíclica, y en segundo lugar de influencias locales.

Todo el sistema consiste en dos zonas de alta presión sobre ambos subtrópicos (una septentrional y la otra meridional) y la franja intermedia de baja presión atmosférica. Hacia esta franja de baja presión convergen las corrientes que se dispersan de los centros de alto nivel, la septentrional y la meridional, forman la línea o zona de convergencia intertropical (ITC).

Debido a la variación de la radiación solar a causa de la translación cíclica (estacional) del sol, se conocen dos posiciones extremas del sistema intertropical (Gráf. 3 y 4 ). La posición meridional al principio del año (febrero) se caracteriza (3) por la división del cinturón meridional de alta presión en doṣ células, interrumpidas sobre el continente suramericano. En consecuencia, las corrientes confluyen hacia la zona de baja presión sobre el interior septentrional del continente, llevando masas ecuatoriales marítimas. La parte norte de la ITC está invadida por masas subtropicales marítimas (Caribe) que constituyen y amplían los centros de alta presión del cinturón septentrional. La posición septentrional se desarrolla paulatinamente cuando masas ecuatoriales marítimas avanzan hacia el norte y refuerzan el cinturón meridional de alta presión cerrándose también sobre $\mathrm{e}$. continente en forma de un puente.

\section{A. EL MACROTIEMPO}

Tal situación en conjunto se denomina el macrotiempo o tiempo general y está regida por la localización de los centros de presión atmosférica que forman el sistema intertropical de circulación y que determinan la dirección y la velocidad de las corrientes actuantes de aire. Con la translación de todo el sistema intertropical cambia paulatinamente la posición de los centros báricos y por consiguiente también las propiedades típicas de las masas de aire arrastradas por las corrientes respectivas. En consecuencia, la ITC cruza también la zona de la vertiente colombiana del Pacífico donde a lo largo convergen masas marítimas o continentales de diferente origen desde direcciones opuestas (generalmente de componentes septentrionales y meridionales, de acuerdo con la diferencia términa principal de los subtrópicos y el Ecuador). Estas corrientes predominantes, conocidas comúnmente como Alisios, hacen converger hacia la ITC masas de diferencias térmicas y de humedad variadas, las cuales ascienden sobre esta zona de acción. La ITC no puede ser una línea o franja fija, como se supone en el sentido climatológico con el concepto de los Alisios, sino que está en permanente movilización por el dinamismo de los procesos atmosféricos. A este respecto es preciso distinguir entre el tiempo reinante momentáneo y el tiempo reinante predominante (estado climatológico). En las latitudes medias tampoco existe permanentemente 
la corriente climatológica occidental, sino que ésta se presenta con la destrucción y el rejuvenecimiento de las perturbaciones atmosféricas y por los cambios de las corrientes de aire; es decir, por cambios del tiempo. De la misma manera, en el trópico también se observan cambios diarios del tiempo, aún durante los períodos de predominio de un carácter del tiempo.

El carácter del tiempo se diferencia en todas partes por valores relativos bien definidos de la radiación y el brillo solar, de las temperaturas y de la humedad del aire, de la nubosidad típica y de la precipitación pluvial. Siempre los días de tiempo anticiclonal (buen tiempo), se caracterizan por la mucha radiación y brillo solar; temperatura relativamente alta; baja humedad del aire, especialmente, en las horas de la tarde; precipitaciones escasas o aguaceros de poca duración. En veces, los díns ciclonales (mal tiempo) se caracterizan por valores contrarios.

Catalogando de acuerdo con las características anotadas los registros semanales (5-11 de marzo y 27 de agosto a 3 de septiembre de 1956) de varias estaciones meteorológicas del occidente colombiano, ordenadas según su latitud, se demuestra visiblemente el transcurso, la translación y la variabilidad del tiempo reinante sobre tal zona (Gráf. 5). Durante el primer período el tiempo ciclonal, con su nubosidad típica aunque con variada intensidad pluvial, avanza desde el sur hacia el norte, debilitándose notablemente al entrar a la zona bajo influencia del centro septentrional alta presión (posición meridional del sistema intertropical); mientras que después de las perturbaciones las cuñas de alta presión del norte se extienden hacia el sur. Así, llegan del norte

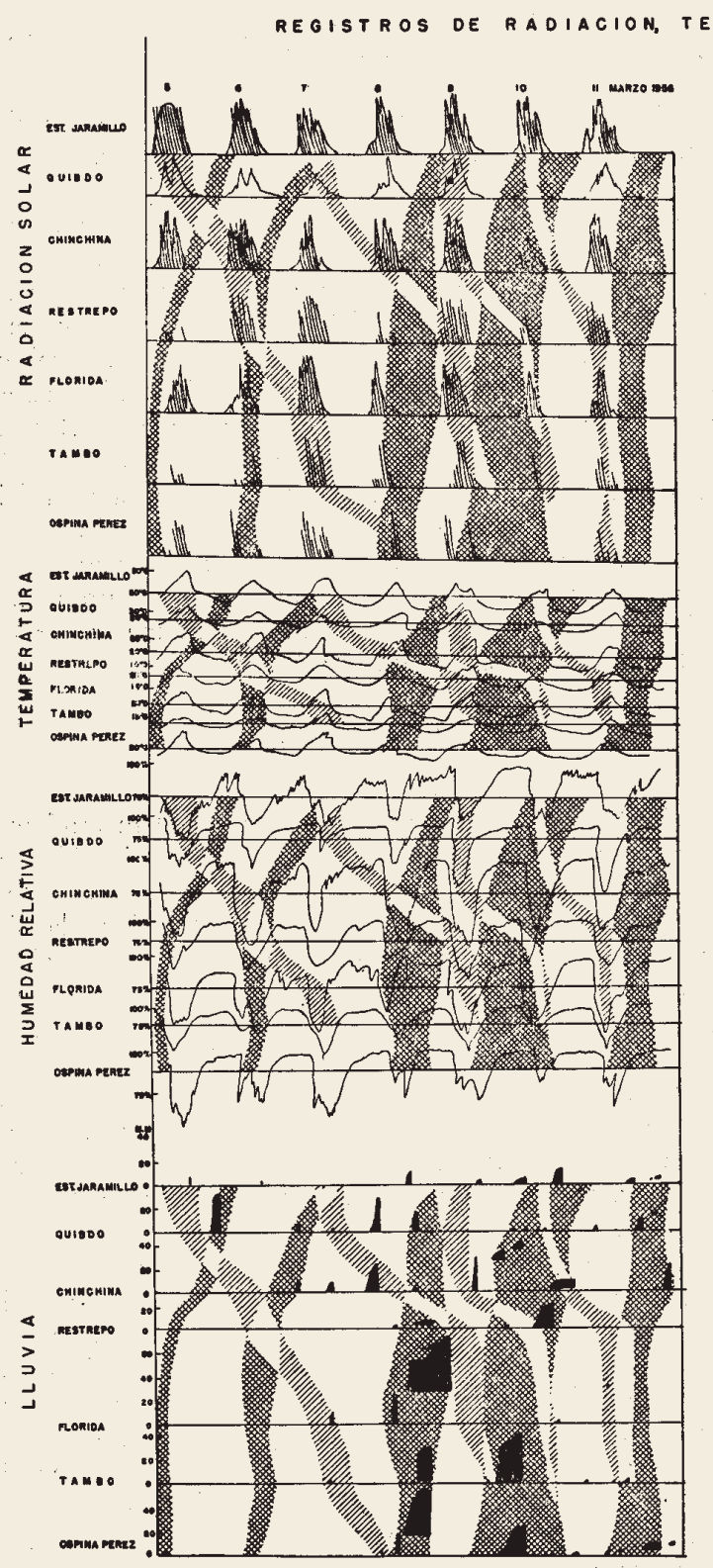

Gurco ins

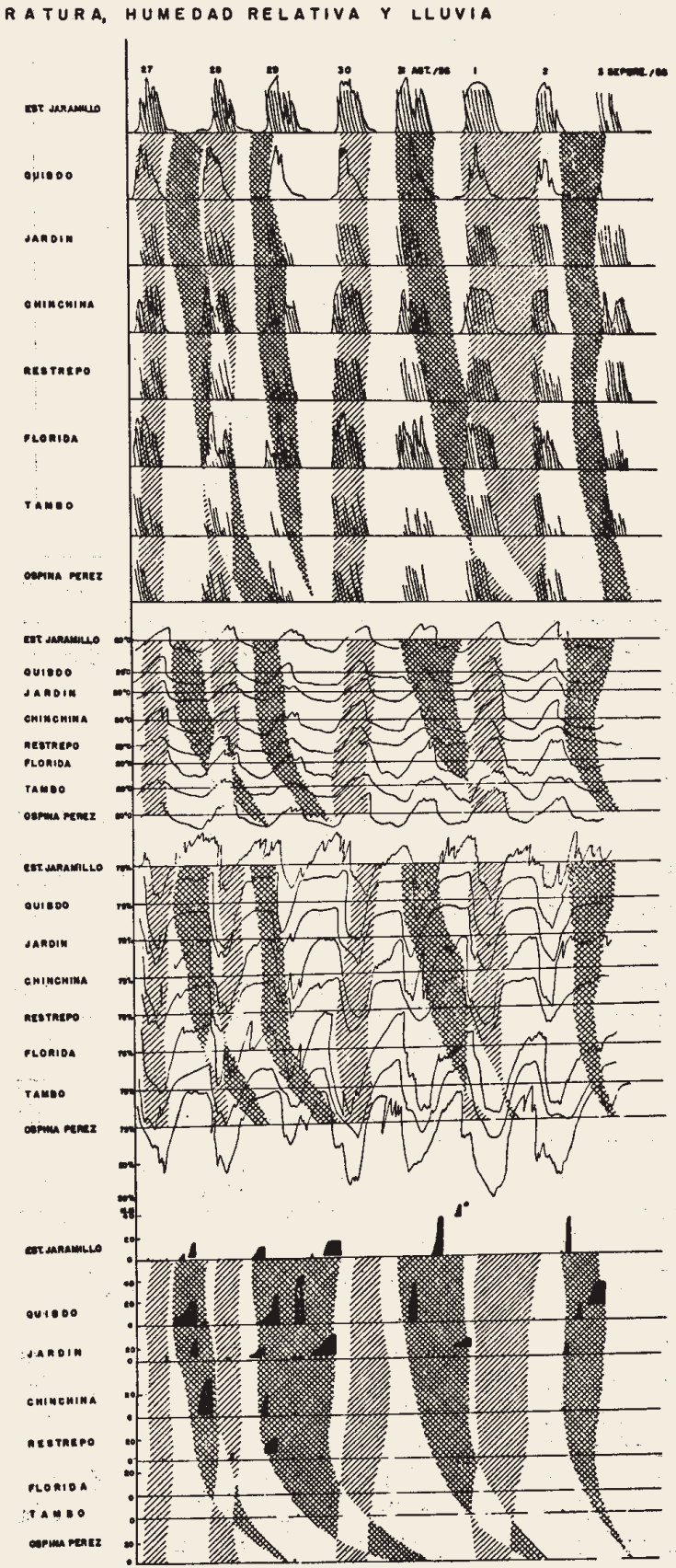


masas de aire fresco del anticiclón del Caribe, las cuales se encuentran hacia el sur con las masas ecuatoriales, y forman nuevas perturbaciones con el mismo traslado general, correspondiente al factor del macrotiempo. Durante el segundo período (27 de agosto a 3 de septiembre de 1956) la translación del macrotiempo es inversa, debido a que el sistema intertropical está en su posición merifional y las perturbaciones atmosféricas se desplazan hacia el sur. El anticiclón meridional se debilita en su actividad, lo cual se manifiesta por el aumento de la nubosidad en los niveles superiores, cambiando transi. toriamente la insolación y caracterizando los demás elementos meteorológicos por actividades de mal tiempo. Las perturbaciones se agotan rápidamente bajo la influencia anticiclonal, extendiéndose las cuñas hacia el norte hasta que se encuentran las masas ecuatoriales con las marítimas subtropicales del Caribe. Allá se forman nuevamente en su línea divisoria las perturbaciones que son empujadas hacia el sur por el aire septentrional fresco.

Según el macrotiempo predominante se definen las épocas estacionales, distinguiéndose principalmente las secas y las lluviosas, resaltando en esta forma un elemento del cual existen generalmente el mayor número de datos. Razones posteriores mostrarán que tal preferencia es puramente artificial y que tal elemento no siempre indica el conjunto como lo manifiesta el tiempo reinante.

Sin embargo, partiendo de lo acostumbrado y conocido, la distribución de los períodos secos y lluviosos se puede generalizar según la forma esquemática dada por Martonne E. (Gráf. 6) de la cual se puede determinar, según la latitud geográfica, la posición cenital del sol y las correspondientes épocas de lluvia y sequía (2). Según esta interpretación entre el Ecuador y los $14^{\circ}$ de latitud existe una doble época anual de Hluvia que, con el aumento de la latitud, se convierte en una sola. A la misma distribución llevan las observaciones pluviométricas representadas según el cuociente de Angot (7) y ordenadas de acuerdo con la latitud, sin considerar la longitud geográfica.

El despazamiento estacional de los períodos secos y lluviosos se puede relacionar con el avance de la ITC lo cual para Colombia ocurre así: La época lluviosa se localiza a pincipios del año en el sur del país y a medida que corre el año avanza hacia el norte, donde impera hasta marzo-abril la época seca. Después de la pri.mera época lluviosa, con duración de dos a tres meses, avanza igualmente de sur a norte y hasta aproximadamente $8^{\circ}$ Lat. N. el tiempo seco. Desde septiembre-octubre, empieza un rápido retorno de las lluvias desde el norte hacia el sur, a través de toda Colombia.

Tal distribución relativa y generalizada se puede encontrar también en todos los datos pluviométricos de la zona en estudio (Gráf. 7) aunque no siempre en forma tan sobresaliente. La altura de la lluvia y con ella su distribución no va siempre en relación directa con el carácter del macrotiempo a lo cual contribuyen además otros factores que se sobreponen hasta predominar en ciertas situaciones atmosféricas como lo demuestran los valores de esta zona excepcional de la vertiente del $\mathrm{Pa}$ cífico (7). •

Sólo una descripción más detallada del desarrollo del macrotiempo permite explicar las desviaciones, lo cual puede realizarse por el estudio de días típicos o por pe- ríodos pronunciados. Los días de buen tiempo y los períodos secos son más pronunciados en los lugares costaneros y en el fondo de los valles amplios. Allí la influencia local (circulación local) refuerza este carácter del tiempo anticiclonal por lo cual sus períodos resaltan en todos los valores relativos (porcientos) de las sumas mensuales de la precipitación, especialmente en los extremos norte y sur (Acandi, Tumaco). En cambio, los períodos lluviosos se destacan más en las zonas altas, donde la circulación local aumenta la actividad ciclonal pluvial. También el efecto de estancamiento y foehn no. se elimina por completo, aun por la representación porcentual, y tampoco las propiedades de estructura de las masas de aire, lo cual se confirma por la distribución de la zona altimétrica media. En esta zona todos los facto-

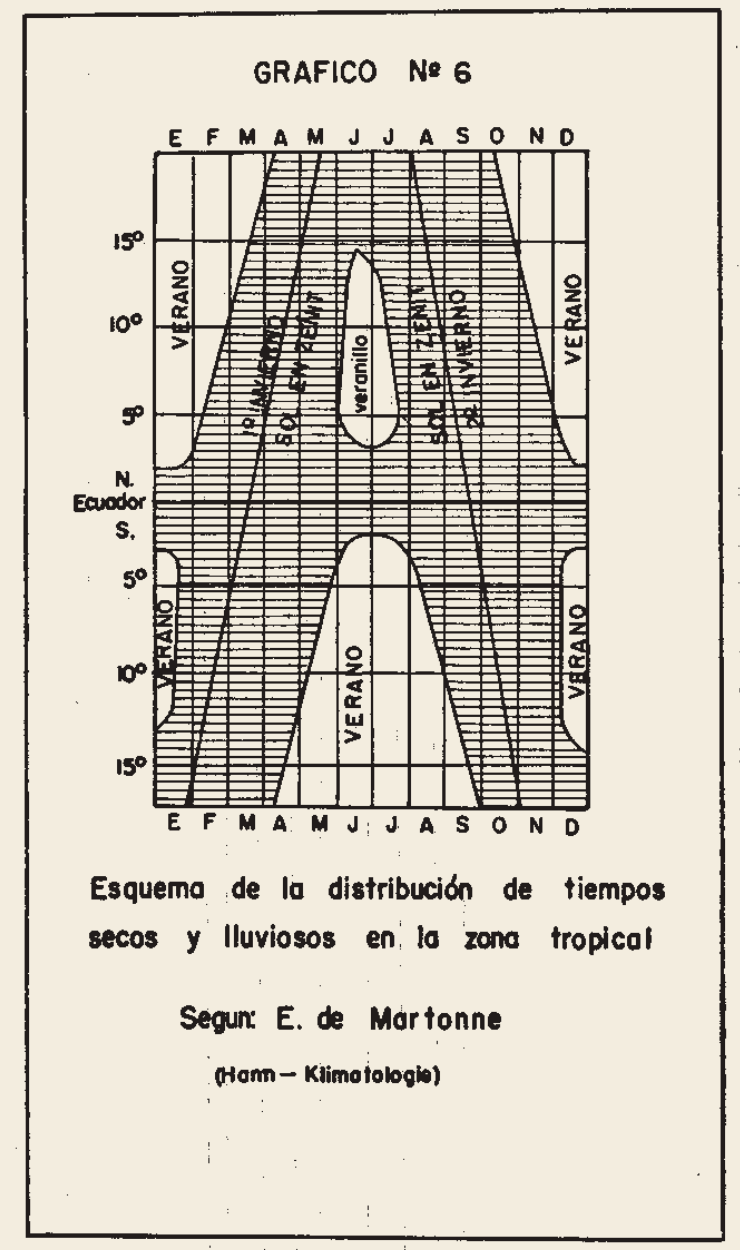

res influyen en la magnitud de la lluviosidad. Las estaciones del norte y del sur (Villa Arteaga, Dabeiba, Consacá, Ricaurte) demuestran el transcurso estacional más pronunciado, como les corresponde según el macrotiempo. No obstante, se observan adelantos y prolongaciones de los tiempos secos y de los lluviosos debido a efectos topográficos, tanto por las corrientes generales (estancamiento, foehn) así como por las circulaciones locales. Las masas de aire marítimo ecuatorial, procedentes del anticiclón meridional del Pacífico avanzan al principio del año arrastradas por las corrientes de WSW y después de SW hacia el centro de baja presión sobre el continente, reforzando por estancamiento las precipitaciones sobre las vertientes occidentales (Ricaurte, Rosas, Anchicayá, Dagua). El efecto del foehn se nota en las esta- 


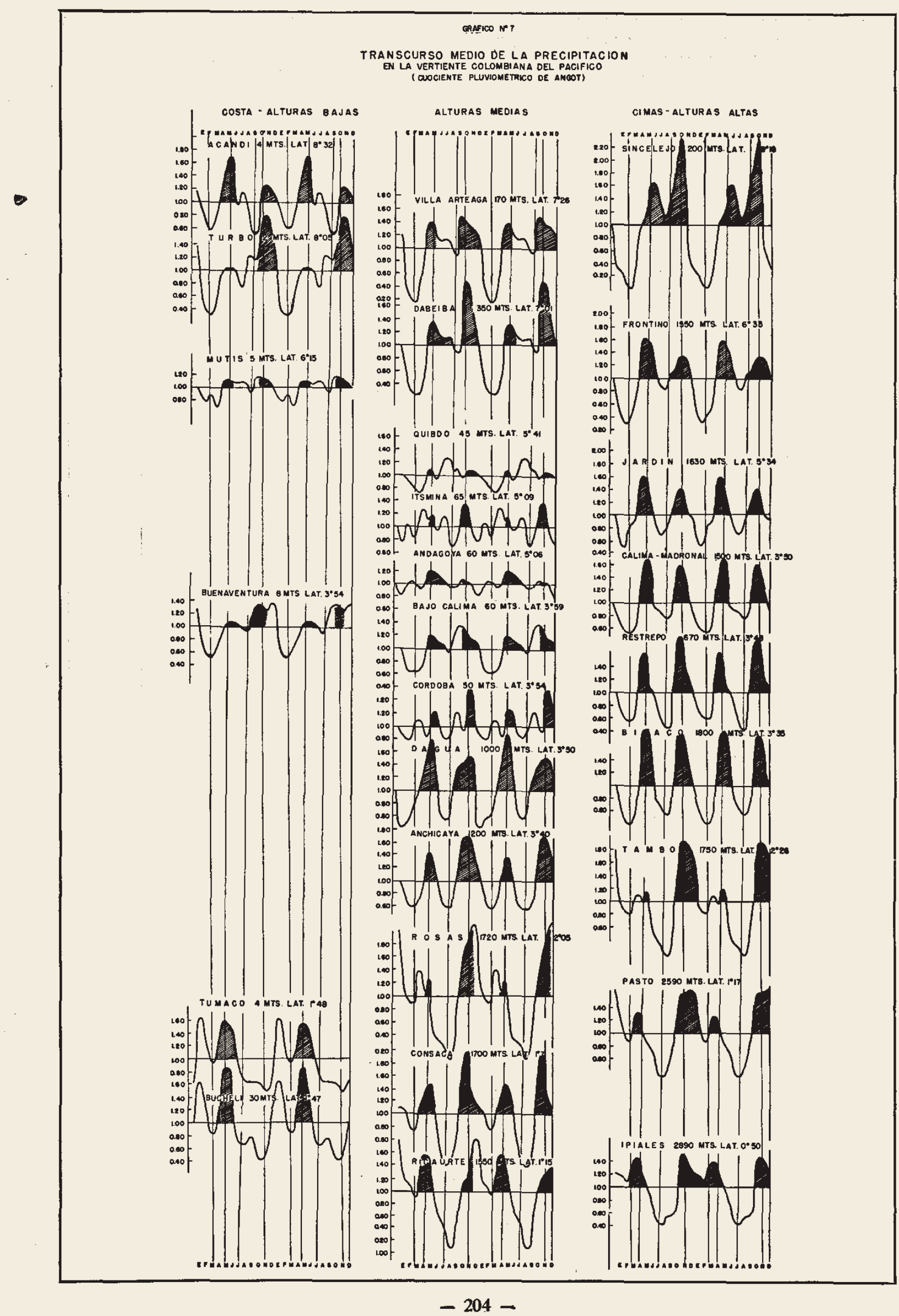


ciones de Pasto y Tambo. Al mismo tiempo, en el norte invaden masas marítimas subtropicales del Caribe del ENE-NE produciendo estancamiento en laderas orientales (Jardín, Restrepo) y el foehn se manifiesta al lado del sotavento (Sincelejo, Calima, Madroñal, Andagoya, Bajo Calima, Córdoba y, aún reconcible, en Rosas).

Siguiendo la translación de la ITC hacia el norte, se extiende el anticiclón meridional el cual forma puente de alta presión sobre la parte norte del continente suramericano; al mismo tiempo, se hace notorio en la parte sur el período seco durante el cual predominan corrientes de direcciones $\mathrm{SE}$ que se manifiesten claramente tanto por la disminución de la pluviosidad como por las características de muy baja humedad y vientos borras. cosos en todas las laderas de sotavento (Pasto, Consacá, Rosas, Tumaco) y más tarde (agosto) también en Córdoba, Bitaco, Calima, Madroñal. Como consecuencia de estancamiento se puede interpretar la prolongación de los aguaceros de mayo.

Cuando empieza a regresar la ITC del norte hacia el sur las corrientes, detrás de las perturbaciones, se voltean primero hacia el NW y después al NE produciendo el estancamiento en las laderas occidentales y orientales respectivamente (Villa Arteaga, Dabeiba, Quibdó). En todo caso, el segundo período lluvioso es generalmente más fuerte en toda la zona debido al mayor contraste térmico de las masas marítimas subtropicales con las ecuatoriales continentales procedentes del puente meridional.

Fácilmente se concluye que no cada año el sistema global de circulación intertropical llega a las mismas posiciones extremas, como demuestran los Anuarios Meteorológicos (14) debido a las fluctuaciones que puede sufrir el sistema de circulación tropical, el cual forma parte de las circulaciones generales de la atmósfera global y por esto también depende de la variada actividad (duración e intensidad) de los demás sistemas, de los cuales son tal vez de mayor importancia los polares y el intertropical. Pues, el macrotiempo que reina sobre estas regiones determina las variaciones de las propiedades de las masas de aire que se forman sobre ellas y que se transmiten también a las otras zonas mundiales. Si durante un año o parte de él, todo el sistema tropical se encuentra relativamente en posición muy septentrional, este lapso se presenta menos lluvioso débido a que la zona de alta presión del cinturón meridional avanza sobre casi todo el territorio de Colombia.

\section{B. LAS CIRCULACIONES LOCALES}

Si los efectos de estancamiento y foehn por la topografía varían según la dirección de las corrientes correspondientes a la distribución bárica (macrotiempo), en las zonas montañosas existen circulaciones locales que evolucionan, de acuerdo con las diferencias térmicas locales producidas por la transformación de la radiación (insolación e irradiación nocturna) que facilita el intercambio de masas de aire, en sistemas verticales cerrados cuya extensión depende de la configuración topográfica y de la estructura vertical del aire. Tales circulaciones se presentan tanto en las zonas costeras como en los valles. Al invertirse las diferencias térmicas se invierte también el sentido de la circulación que se desplaza siempre en sus niveles inferiores desde la zona fría hacia la caliente donde asciende el aire y se enfría termodiná- micamente. Sobre las zonas de ascenso, el enfriamiento provoca la condensación y por consiguiente la formación de nubosidad y de precipitaciones; mientras que en la parte descendente las nubes se disuelven.

Naturalmente, como la estructura vertical de las masas de aire cambia de acuerdo con el macrotiempo, las circulaciones locales se desarrollan diferentemente durante cada situación estacional. Sin embargo, debido a la acción permanente y proporcional durante todo el año, la caracterización de cada lugar respecto a su ubicación topográfica se refleja aun en los valores medios anuales de todos los elementos. Además, según cada configuración típica de la topografía, la circulación local imprime manifestaciones meteorológicas muy determinadas que se diferencian en todos los valores de los elementos. Estas variaciones se conocen con el concepto del ciclo diario de cada elemento meteorológico el cual es causado principalmente por el ciclo solar diario y por el carácter del tiempo como función del macrotiempo. Debido a su desarrollo claro y regular, son suficientemente conocidas las características esenciales del ciclo diario para la mayoría de los elementos meteorológicos; por ejemplo, la onda doble de la presión atmosférica y el ciclo bien definido de la temperatura del aire, de la humedad relativa, de la radiación y del brillo solar en forma de onda simple (Gráf. 8). Aunque se aprecia claramente una variación estacional del ciclo diario como reacción del macrotiempo con sus masas actuantes, las características de cada elemento se mantienen firmes. Desde luego, las singularidades más típicas se observan durante días de buen tiempo o durante períodos anticiclonales, cuando el ciclo diario de los elementos meteorológicos es más pronunciado y por lo tanto las circulaciones locales mejor desarrolladas.

De lo anterior se concluye, tal como se ha confirmado para los Alpes por observaciones durante buen tiempo, que las diferencias notables del ciclo diario de los elementos en zonas en el fondo de valles, en laderas y en cimas dependen, de su parte también, de las circulaciones locales, las cuales modifican los ciclos de los diversos elementos como reacción de la radiación y de su transformación calórica en la superficie terrestre. En todo caso, en los valles y en las cimas se registran oscilaciones más marcadas que en las laderas y, por otra parte, la desviación del promedio diario es contraria en los niveles extremos. Las máximas sobresalen en los valles más que el valor promedio en las cimas y las mínimas bajan más en las cimas que en los valles lo cual es válido para todos los elementos que tienen el mismo ciclo que la radiación y que la temperatura; en contraste, la humedad tiene un ciclo inverso. El ciclo diario durante el tiempo ciclonal es proporcionalmente menos pronunciado, y puede transfigurarse, también en el trópico, por características del cambio de masas de aire.

Para la temperatura, la cual correlaciona la densidad y la humedad del aire, el gráfico siguiente (Gráf. 9) muestra las variaciones correspondientes a la diferente ubicación de la estación meteorológica. En las laderas altas, la reducción de la insolación por la nubosidad local, como consecuencia del ascenso de la circulación diurna, disminuye las temperaturas máximas; y en las partes bajas de las laderas el aumento del contenido de vapor acuoso disminuye la irradiación nocturna y el enfriamiento. También el ascenso térmico en la mañana y el descenso de la tarde reflejan la ubicación y el efecto de la nubosidad local. 


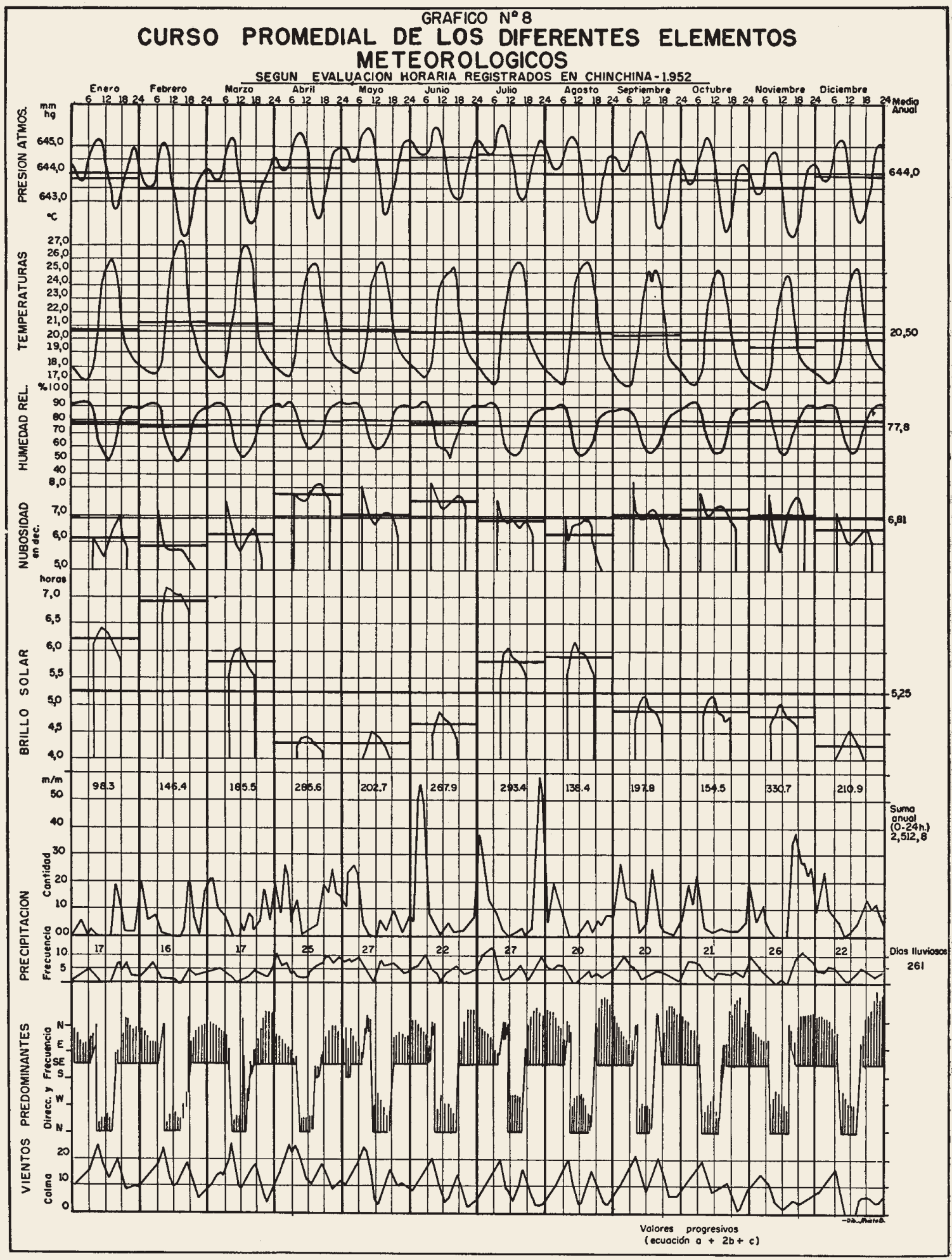


GRAFICO $\mathrm{N}^{\circ} \mathrm{g}$

\section{CONTRASTES DEL CICLO DIARIO \\ DE LA TEMPERATURA \\ DURANTE BUENO Y MAL TIEMPO}

EN DIFERENTES LOCALIDADES TIPICAS
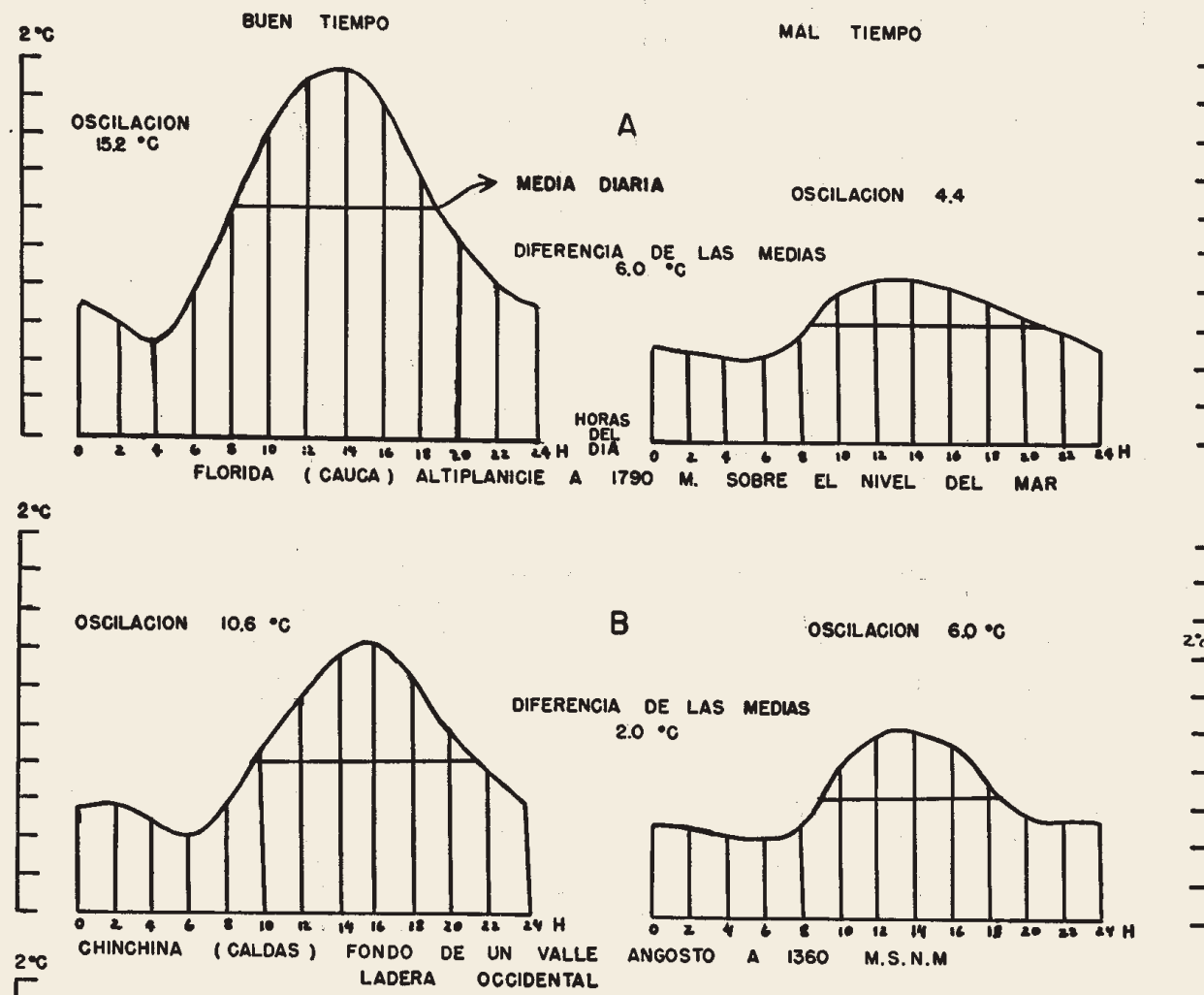

B OSCILACION $6.0^{\circ} \mathrm{C}$
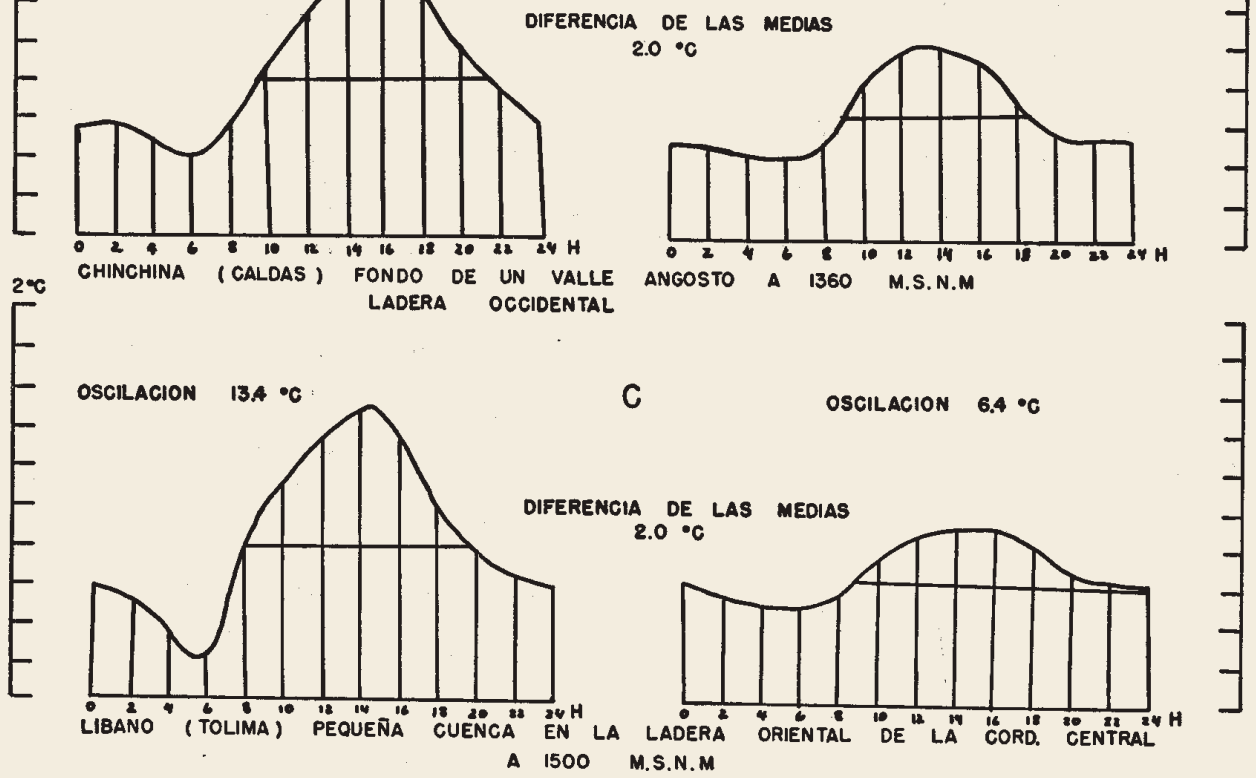

5
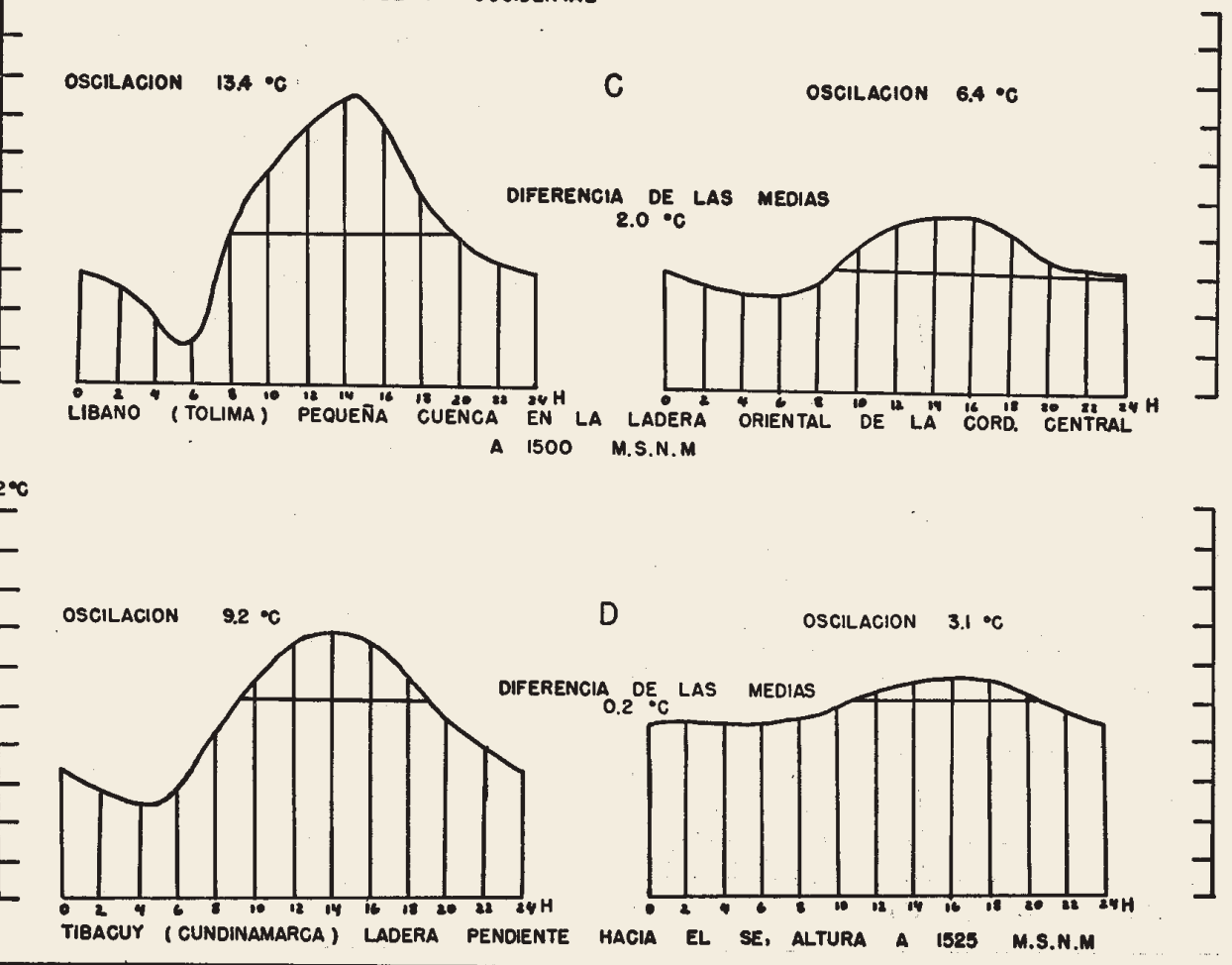

$-1$ 


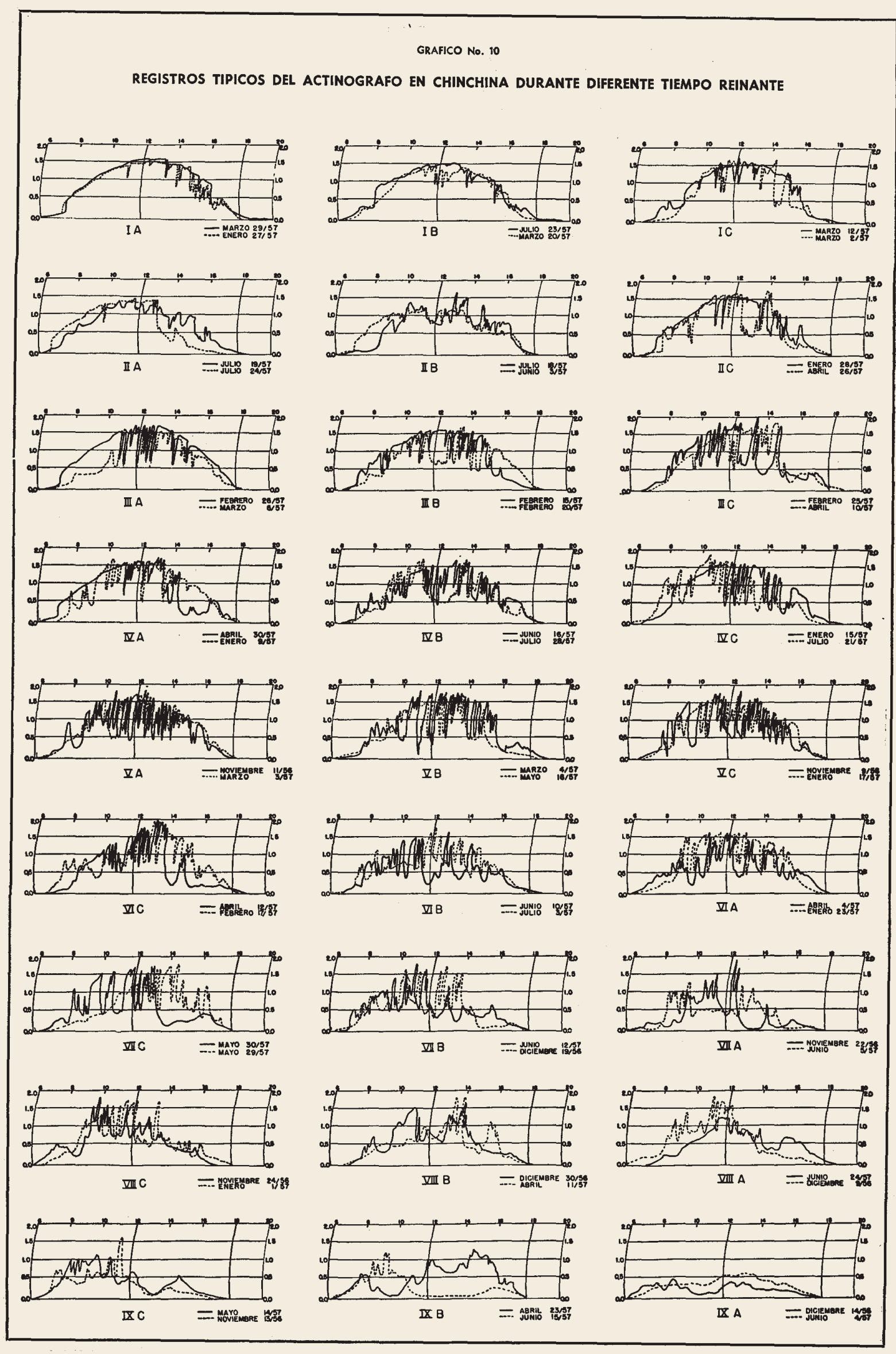


La variación de la radiación diaria (fuente de energía para las circulaciones locales) se presentan en los registros actinográficos, de los cuales los diferentes tipos manifiestan la influencia de la nubosidad según el tiempo reinante, p.e. en Chinchiná (Gráf. 10) los registros I a III corresponden a días de buen tiempo y los VIII y IX para días nublados y lluviosos, mientras que los otros representan días con nubosidad variable. La cantidad calórica (gr. cal $/ \mathrm{min} . \mathrm{cm}^{2}$ ) recibida por una superficie horizontal alcanza su máxima desde 9-10 horas hasta 1516 horas en cielo despejado y durante tiempo nublado es inversa a la densidad de la nubosidad; estos registros reflejan bien el carácter del tiempo sobre cada lugar. Los valores altos durante el buen tiempo contrastan con los valores bajos durante días lluviosos, en los cuales disminuye la radiación a una quinta parte aproximadamente. Además, la forma de la curva registrada indica notablemente la variabilidad de la nubosidad.

La interpretación del ciclo diario de la precipitación (Gráf. 11) muestra claramente la influencia de las circulaciones locales y su relación con la ubicación topográfica del lugar. Como es sabido, se conoce una circulación diurna del valle hacia la montaña; cuando el aire asciende, forma las nubes de desarrollo vertical sobre las partes altas y las cimas. De allí se desprenden las precipitaciones y toman rumbo hacia el valle, con la corriente superior de regreso, cubriendo una franja hasta de una tercera parte de la altura relativa. El nivel del techo de estas nubes depende del grado de saturación del aire en el fondo del valle, donde el volumen empieza su movimiento de ascenso. Por esto, el techo se levanta en las horas de la mañana (nieblas de laderas que se levantan y se disuelven). Cuando las circulaciones alcanzan su pleno desarrollo, el nivel de la nubosidad se forma a determinada altura sobre el fondo de los valles (nivel de condensación), pero esta relación disminuye con el aumento de la altitud, observándose un nivel general algo inclinado hacia las cimas.

La distribución horaria relativa de la precipitación muestra una acumulación de la lluvia (cantidad, frecuencia, densidad e intensidad) en las horas de la tarde como lo indica notoriamente la estación de Restrepo. Tanto las sumas horarias anuales de la precipitación, como su combinación en intensidad (cantidad-duración) y frecuencia, presentan estas características. Aun mejor resalta este ciclo diario en la distribución porcentual del factor combinado durante los meses menos lluviosos (secos) como junio, julio y agosto durante los cuales la precipitación se debe principalmente al efecto de la circulación local diurna. La circulación contraria, la cual se desarrolla cuando el aire frío de la montaña fluye ha. cia el valle donde asciende, produce las lluvias predominantes de la noche (Quibdó, Bajo Calima) lo cual confirman también algunas observaciones generales (1). Según la distribución del "factor de combinación" se aprecian dos máximas las cuales se pueden interpretar como efecto producido por olas de aire frío. Después de la primera, las mismas precipitaciones ocasionan la estabilización posterior durante la cual cesa la pluviosidad, para revivir el proceso tres o cuatro horas más tarde. La importancia que tiene este fenómeno local en las sumas mensuales, especialmente en las zonas húmedas tropicales, se puede tomar en consideración comprobando los altos valores de lluvia durante los meses de junio, julio y agosto que se presentan en esta zona (Quibdó: junio $860,1010,960 \mathrm{~mm}$. Bajo Calima: 640, 730, $710 \mathrm{~mm}$. res- pectivamente) fuera del transcurso estacional normal. La zona intermedia, donde actúan ambas circulaciones locales, la diurna y la nocturna, se caracteriza por la mayor pluviosidad (8) y la distribución horaria relativa tiene dos máximas (Dosquebradas). Según la localización del lugar predomina el uno o el otro sistema de circulación, pero el nocturno se destaca generalmente más (7) por cuanto está reforzado por el levantamiento topográfico (estancamiento) al trasladarse las precipitaciones con la corriente superior desde el valle hacia la montaña (Chinchiná). La renovación de las circulaciones, tanto diurnas como nocturnas, se reconoce en las máximas secundarias; las precipitaciones en laderas entre las 8-9 horas corresponden al primer levantamiento del aire matinal al comienzo de las circulaciones diurnas, y las del mediodía se deben interpretar como las primeras de las precipitaciones diurnas.

Las circulaciones locales se manifiestan naturalmente, también, por los registros de los vientos superficiales y por su ciclo diario. Después de la calma matutina comienzan los vientos a ascender a lo largo del fondo de los valles (Talaufwind) para luego extenderse hacia las vertientes (Hangaufwind) y finalmente amplificarse el ascenso por todas las laderas lo cual se denomina viento del valle (Wagner). En las horas vespertinas, al cesar el calentamiento por la insolación, o cuando se cubre con nubes todo el valle, se observa un corto período de calma y luego el viento voltea a la dirección opuesta (vientos de la montaña), acompañado frecuentemente con ráfagas (olas de aire frío). Los vientos predominantes de todas las observaciones están de acuerdo con los correspondintes a las circulaciones locales (7); así se manifiesta en los promedios según el horario de las observaciones. En consecuencia, las circulaciones locales son características muy destacadas en todas las zonas húmedas montañosas donde predomina durante el año la inestabilidad de las masas de aire.

\section{DATOS CLIMATOLOGICOS DE LAS ZONAS DE. LA VERTIENTE COLOMBIANA DEL PACIFICO}

Si ya es difícil la descripción detallada del clima de las regiones más pobladas de Colombia con base en datos relativamente completos, tal estudio se hace más difícil para la zona húmeda del Pacífico. Hasta el presente, toda la atención climatológica se concretó a recoconocer tal región como una de las zonas más lluviosas del mundo, sin mayor preocupación por sentar las bases de observación indispensables para un estudio climatológico detallado, con lo cual se llegó al resultado de que el carácter climatológico excepcional de esta zona más bien sirvió de obstáculo que de aliciente para su estudio.

En las condiciones actuales, es difícil que por medio de una interpretación dinámica se pueda obtener una descripción completa; sin embargo, aprovechando los datos existentes y los de regiones adyacentes, se intentará destacar las zonas de necesidad inmediata para realizar observaciones meteorológicas, que permitan comprobar adecuadamente la interpretación preliminar.

Para cualquier lugar de la región, los valores meteorológicos característicos dependen del carácter del tiempo reinante según su trascurso estacional y de las modificaciones locales. Del macrotiempo dependen las propiedades subjetivas de las masas de aire; y del cambio de ellas depende, también, la variación del carácter del 
GRAFICO $\mathrm{N}^{2}$ II

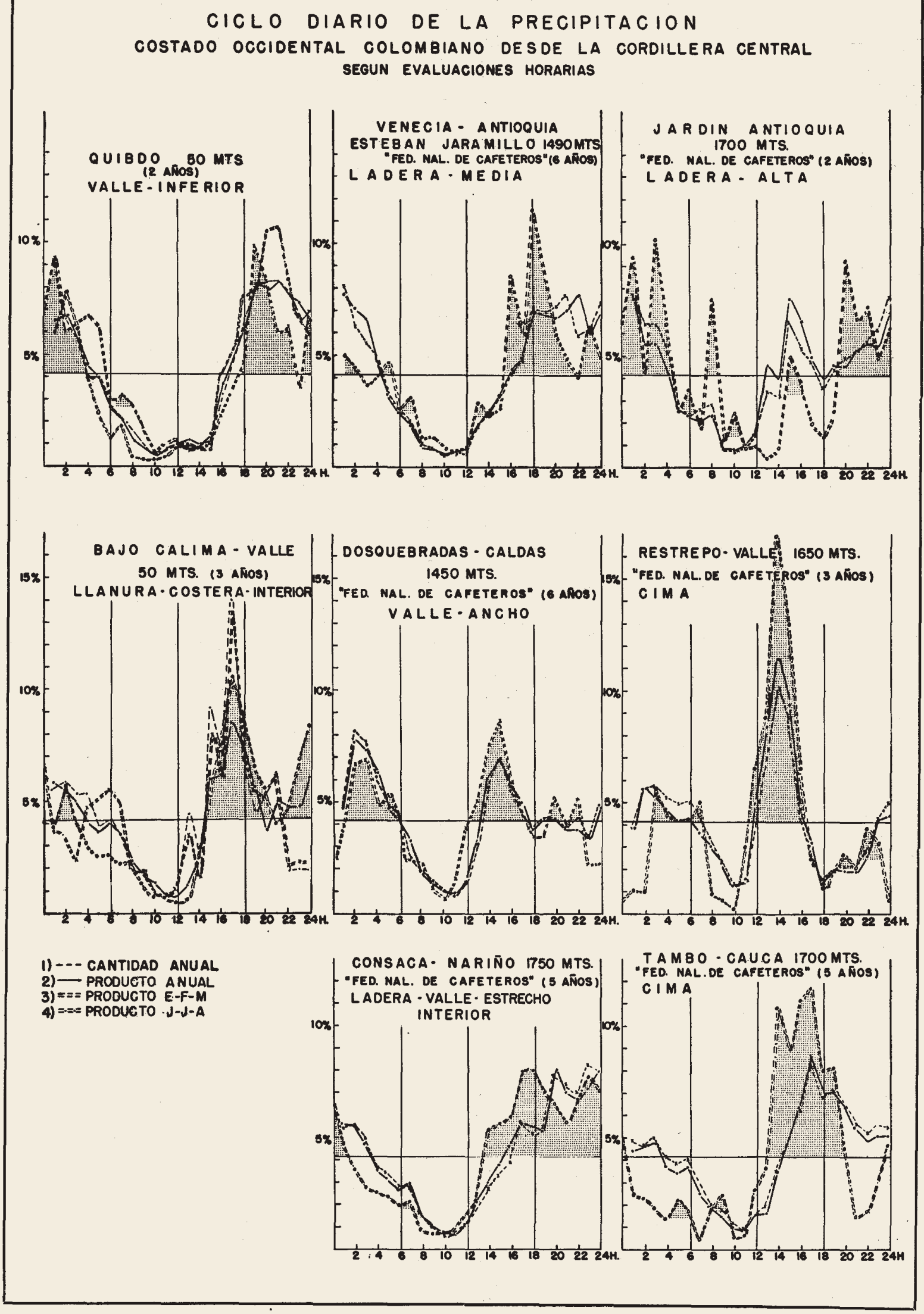


tiempo diario y hasta el carácter general de ciertos lapsos de tiempo diario semejantes. Es preciso encontrar las diferencias más notorias que se muestran en las variaciones de los tipos principales de tiempo (anticiclonal y ciclonal). Naturalmente, la adición de estas situaciones individuales con sus valores respectivos a sumas mensuales y anuales, y su promedio disminuye aparentemente las diferencias hasta que no se observan características estacionales marcadas en la mayoría de los elementos meteorológicos. Además, las propiedades características de las masas de aire que actúan en el desarrollo del tiempo no pueden diferenciarse en tal grado como en las latitudes medias, donde debido a la variación estacional de la radiación (posición solar) ocurren adicionalmente cambios más fuertes de las masas de aire advectivas (polares y tropicales). Sin embargo, es de gran importancia para el estudio del clima local y aún mundial la atención que se debe prestar a la zona de formación de las masas de aire tropicales y ecuatoriales.

Cada una de las masas que son transportadas por las corrientes generales tienen, según su definición y catalogación, características específicas en su contenido calórico y de humedad. Las masas ecuatoriales son calientes y se trasladan del Ecuador térmico hacia los subtrópicos, conservando su calor en toda la trayectoria; las masas subtropicales se presentan en todos los niveles más frescas; mientras que las denominaciones de marítimo y continental determinan el grado de saturación con vapor acuoso. También la disminución de la temperatura y de la altura del nivel de condensación varía de acuerdo_con las características mencionadas de las diferentes masas de aire. La disminución altimétrica de la temperatura hasta alcanzar el nivel de condensación es de $1^{\circ} \mathrm{C} / 100 \mathrm{~m}$., nivel que cambia durante el día según la variación de la humedad de la superficie. A partir del nivel de condensación la disminución térmica altimétrica se reduce a $0,5^{\circ} \mathrm{C} / 100 \mathrm{~m}$. Estas dos condiciones determinan el nivel básico de la temperatura de cualquier lugar al cual se adiciona el calentamiento local según la radiación efectiva. En consecuencia, la disminución de la temperatura con la altura es más lenta en las zonas húmedas que en el interior (aire menos húmedo). La alta humedad del aire reduce también notoriamente la radiación general, según la ubicación del lugar respecto a la influencia de circulaciones locales. Esta reducción de las condiciones de radiación se refleja también en una oscilación muy pequeña de la temperatura alrededor de las medias anuales de 25 a $27^{\circ} \mathrm{C}$ a nivel del mar (Buenaventura, Tumaco) y un ligero aumento en el fondo de los valles del río Atrato y del San Juan a 26 a $28^{\circ} \mathrm{C}$ (Quibdó) y especialmente en las partes bajas de la cuenca del río Patía, debido al ligero aumento de la insolación que hace más calientes los valles que otras localidades de las mismas alturas, aun en las zonas húmedas. Además, la variación de las condiciones térmicas se muestra en una pequeña oscilación diaria $\left(3\right.$ a $4^{\circ} \mathrm{C}$ ) durante mal tiempo; y 8 a $10^{\circ} \mathrm{C}$ durante buen tiempo; esta oscilación es aun menor en lugares de vertientes debido a efectos topográficos locales. Tomando en cuenta estos puntos de vista (el macrotiempo y la modificación local) se pueden caracterizar con bastante precisión las condiciones térmicas de cada lugar), aun con mayor facilidad cuando se conocen por medición directa los gradientes térmicos para los diferentes tipos del tiempo y su transcurso anual.
El conocimiento del estado térmico exige la relación estrecha con la humedad del aire, de acuerdo con la relación física entre temperatura y humedad. La humedad relativa media anual en casi toda la zona es de más del $85 \%$; y las mínimas de la tarde no bajan aun en los valles y durante buen tiempo a $45 \%$ (Chinchiná $30 \%$ ) como se comprueba por los registros automáticos de Quibdó y del Bajo Calima (4). La característica más acentuada de las zonas húmedas es naturalmente su dependencia de la humedad absoluta $(\mathrm{gr} . / \mathrm{m} 3)$ o de la tensión del vapor $(\mathrm{mm}$. Hg.). La tensión del vapor muestra valores altos, especialmente en la zona costera y en el fondo de los valles con temperaturas anuales de 26 a $28^{\circ} \mathrm{C}$ y un promedio de la humedad relativa de 80 a $90 \%$, al cual corresponde una tensión media de 22 a $24 \mathrm{~mm}$. $\mathrm{Hg}$. cuando más de 16 a $18 \mathrm{~mm}$. se considera en las latitudes medias como sofocante para el hombre. Sin embargo, en los días lluviosos (frescos) con baja tensión del vapor (p. e. $20^{\circ} \mathrm{C}, 100 \%, 18 \mathrm{~mm}$. $\mathrm{Hg}$.), como en días de buen tiempo $\left(33^{\circ} \mathrm{C}, 50 \%, 18 \mathrm{~mm}\right.$. $\mathrm{Hg}$.) el habitante aclimatado no sufre el aire sofocante; además, una ligera circulación del aire reduce temporalmente también este efecto desagradable de las zonas húmedas. Por el contrario, en tiempos de nubosidad variable con temperaturas menores pero alta humedad $\left(29^{\circ} \mathrm{C}, 75 \%\right.$, $22,9 \mathrm{~mm}$. Hg.), especialmente al cubrirse el cielo con nubes translúcidas, el ambiente se hace extremadamente sofocante. La tensión del vapor disminuye con la altitud de acuerdo principalmente con la disminución de la temperatura, en tal forma que por encima de los 1000 m. desaparece el ambiente pesado apesar de la alta humedad relativa casi permanente.

Un buen índice de las características climáticas de cada lugar es el brillo solar, cuya duración manifiesta las condiciones locales y el tiempo reinante. Por la eliminación del efecto puro de la topografía, la pérdida variable por los alrededores durante las horas de la mañana y de la tarde, resulta la duración efectiva, la cual se determina teóricamente por un levantamiento topográfico del horizonte o empíricamente con base en observaciones de varios años. El conocimiento de este valor permite deducir la reducción real por acción de la nubosidad; sin embargo, las diferencias más notorias por la variación del macrotiempo y por la influencia de las circulaciones locales se obtienen simplemente por los datos comunes. La región del Bajo Calima (llanura costera interior) recibe aproximadamente sólo la mitad de la insolación que Palmira en el Valle del Cauca, conservándose el transcurso general del macrotiempo en ambas regiones. El aumento del brillo solar durante los meses de julio-agosto por la influencia anticiclonal resalta mejor por estos datos que por la distribución pluvial en la cual se observan sumas altas durante este período. Aunque las fluctuaciones de la duración porcentual del brillo solar son menores, estas representan más claramente la distribución generalizada según los datos pluviométricos. La relación altimétrica como influencia de las circulaciones diurnas también se refleja como puede verse en los datos de la tabla 1 . En el corte geográfico Bajo Calima (40 m.) 1182 horas, Restrepo (1590 m.) 1815, Palmira $(1080 \mathrm{~m}$.) 2232, Calarcá $(1500 \mathrm{~m}$.) 1404, y Sevilla $(1550 \mathrm{~m}$.) 1326 horas, se reconoce la disminución de la duración del brillo solar debido a la presencia de la nubosidad diurna. Durante el período seco (junio, julio y agosto) los valores respectivos son: Bajo Calima 338, Restrepo 504, Palmira 592, Calarcá 406 y Sevilla 434 horas, de lo cual se concluye que la reducción del 
brillo solar ocurre notablemente a partir del segundo tercio de altitud relativa (Cordillera Central, Vertiente Occidental fondo $1000 \mathrm{~m}$., cimas generales $3.500-4.000$ m., nivel de condensación 1800-2000 m.) cuyas nubes se extienden hacia el Valle hasta la zona de $1400 \mathrm{~m}$.; en analogía en la Vertiente del Pacífico (nivel del mar, cimas 2200-2500 m., nivel de condensación 500-700 m.) la influencia de las nubes respecto a la disminución notoria del brillo solar alcanza hasta 150 a $200 \mathrm{~m}$. Las pro- pias cimas sufren durante tiempo anticiclonal una reducción menor, debido al carácter de la circulación diurna, cuya parte superior va dirigida hacia los valles, y al secamiento de las capas superiores de la atmósfera por efectos de subsidencia.

La reducción más frecuente se observa, también, de acuerdo con el ciclo diario de la precipitación, después del medio día, lo cual se confirma según la frecuencia media de horas sin sol (tabla $\mathrm{N}^{9} 2$ ) donde se muestra

TABLA No 1 BRILLO SOLAR

\begin{tabular}{|c|c|c|c|c|c|c|c|c|c|c|c|c|c|c|c|c|}
\hline ESTACION & Lat. & Alt. & Mts. & Ene. & Feb. & Mar. & Abr. & May. & Jun. & Jul. & Ago. & Sep. & Oct. & Nov. & Dic. & Total \\
\hline Galerazamba & $10^{\circ}$ & $47^{\prime}$ & 3 & 265 & 261 & 252 & 215 & $\mid 93$ & 181 & 216 & 199 & $183 \mid$ & 160 & 195 & 229 & 2549 \\
\hline Venecia & $5^{\circ}$ & $55^{\prime}$ & 1450 & 192 & 184 & 183 & 132 & 170 & 194 & 228 & 232 & 166 & 112 & 141 & 154 & 2549 \\
\hline Jardín & $5^{\circ}$ & $34^{\prime}$ & 1630 & 191 & 210 & 200 & 154 & 150 & 192 & 209 & 201 & 174 & 120 & 137 & 159 & 2097 \\
\hline Manizales & $5^{\circ}$ & $04^{\prime}$ & 2150 & 194 & 180 & 156 & 140 & 133 & 147 & 195 & 172 & 137 & 99 & 135 & 137 & 1825 \\
\hline Chinchiná & $4^{\circ}$ & $58^{\prime}$ & 1360 & 196 & 160 & 159 & 119 & 129 & 137 & 186 & 184 & 155 & 131 & 139 & 162 & 1857 \\
\hline Santa Rosa de Cabal & $4^{\circ}$ & $51^{\prime}$ & 1470 & 167 & 155 & 148 & 134 & 117 & 145 & 162 & 151 & 146 & 116 & 144 & 157 & 1742 \\
\hline Calarcá & $4^{\circ}$ & $31^{\prime}$ & 1500 & 141 & 126 & 114 & 96 & 100 & 123 & 146 & 137 & 115 & 83 & 100 & 123 & 1404 \\
\hline Sevilla & $4^{\circ}$ & $17^{\prime}$ & 1550 & 119 & 106 & 102 & 88 & 95 & 124 & 148 & 162 & 130 & 72 & 85 & 95 & 1326 \\
\hline Bajo Calima & $4^{\circ}$ & $00^{\prime}$ & 40 & 106 & 90 & 89 & 88 & 95 & 98 & 121 & 119 & 94 & 90 & 85 & 107 & 1182 \\
\hline Restrepo & $3^{\circ}$ & $50^{\prime}$ & 1590 & 174 & 151 & 151 & 138 & 152 & 155 & 167 & 182 & 158 & 127 & 129 & 131 & 1815 \\
\hline Palmira & $3^{\circ}$ & $32^{\prime}$ & 1080 & 205 & 196 & 188 & 166 & 169 & 182 & 203 & 207 & 184 & 170 & 172 & 190 & 2232 \\
\hline Popayán & $2^{\circ}$ & $26^{\prime}$ & 1790 & 165 & 147 & 134 & 105 & 117 & 130 & 169 & $167 \mid$ & 146 & 95 & 120 & 134 & 1629 \\
\hline Tambo & $2^{\circ}$ & $36^{\prime}$ & 1750 & 165 & 160 & 136 & 133 & 101 & 163 & 186 & 183 & 139 & 117 & 131 & 169 & 1783 \\
\hline Consacá & $1^{\circ}$ & $17^{\prime}$ & 1700 & 140 & 134 & 121 & 109 & 127 & 154 & 177 & 191 & 149 & 83 & 135 & 122 & 1642 \\
\hline
\end{tabular}

que entre las 11 y las 14 horas aumenta la nubosidad local con alguna anticipación a la frecuencia de la precipitación, después de lo cual se despeja rápidamente el cielo. Esta nubosidad reduce la duración del brillo solar a la máxima de 6-8 horas diarias (Bajo Calima) también durante los períodos de buen tiempo. Sin embargo, ocasionalmente se presentan tardes con 5 horas de brillo solar, debido al despejamiento rápido por subsidencia en la atmósfera superior.

Sobre la reducción de la radiación y la variación de la distribución espectral causada por la absorción debida al alto grado de vapor de agua en la atmósfera, no existen todavía datos; pero por estimación y comparación con otras regiones (10), se puede suponer una reducción por efecto de nubes densas a menos de una quinta parte del valor correspondiente a cielo despejado (Gráf. 12) lo cual se confirma según observaciones fotométricas realizadas en Quibdó. La falta de observación de la radiación es muy lamentable y solo explicable por el costo y complicado manejo de estos aparatos, apesar de la gran importancia de este factor meteorológico sobre la vida en general por su influencia en todos los procesos biológicos.

La cantidad pluvial en las zonas tropicales y también en las húmedas no es estrictamente proporcional con el carácter del tiempo ciclonal (9). Los aguaceros, forma predominante de las lluvias tropicales, no siempre poseen sus intensidades más altas durante tiempo ciclonal, por cuanto el factor de las circulaciones locales es reducido por la falta de fuertes diferencias térmicas locales. Por esto, para una cabal interpretación de la precipitación se hacen necesarias otras características tales como la duración, la frecuencia, la intensidad, las horas de iniciación y el rumbo de las lluvias. La diferente distribución de estas características se muestra en el gráfico 13 con base en datos de Chinchiná. Evaluaciones de regis-

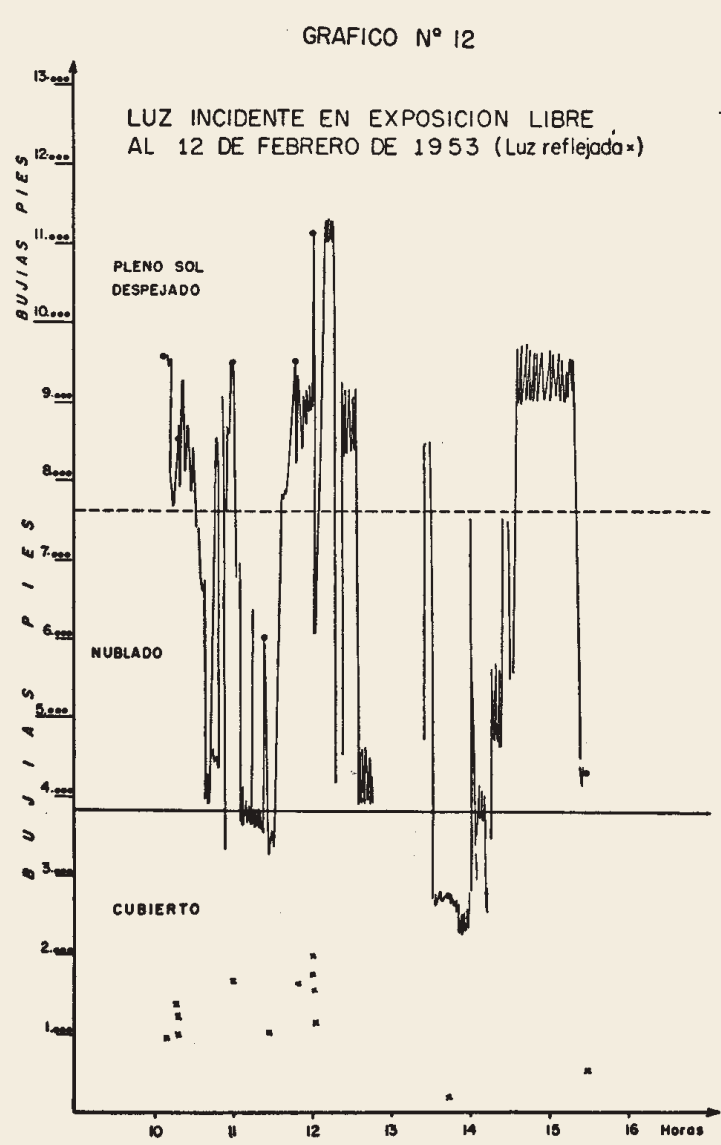

tros pluviográficos dan como valores básicos: la cantidad, la frecuencia $(H)$ y la duración horaria y su cómputo las sumas mensuales y anuales correspondientes; de 
estos se deducen algunas características pluviales como la densidad media (cantidad/frecuencia) y la intensidad (cantidad/duración). Por la multiplicación de intensidad por frecuencia (I x H) resulta un producto que combina los elementos básicos en una "cantidad calculada". E] transcurso anual y el transcurso diario son en grandes rasgos el mismo para los elementos básicos y para el producto, pero varía considerablemente respecto a la densidad y a la intensidad. En el transcurso anual la máxima de la intensidad y de la densidad según la distribución porcentual, se observa en Chinchiná durante el mes de marzo (período de transición) disminuyendo durante los períodos lluviosos y secos; mientras que las otras épocas de transición se manifiestan solo por muy pequeñas máximas secundarias. Este hecho se puede interpretar como resultado de la mayor inestabilidad de las masas marítimas ecuatoriales así como de condiciones más favorables durante esta época para el desarrollo de circulaciones locales como lo indican los valores respectivos (tabla No 3), los cuales corresponden al ciclo característico de este lugar, según la cantidad horaria anual y la del producto $(\mathrm{I} \times \mathrm{H})$ durante el período seco de los meses de enero, febrero y marzo.

\section{TABLA No 2}

Frecuencia mensual de horas sin sol y suma mensual del brillo solar para el mes de agosto, estación Restrepo:

\section{HORAS RADIACION MAXIMA}

\begin{tabular}{|l|c|c|c|c|c|c|c|c|c|c|}
\hline \multicolumn{1}{|c|}{ HORAS } & $7-8$ & $8-9$ & $9-10$ & $10-11$ & $11-12$ & $12-13$ & $13-14$ & $14-15$ & $15-16$ & $16-17$ \\
\hline Frecuencia & 12 & 5 & 21.2 & 21.1 & 17.9 & 14.8 & 16.2 & 20.8 & 20.7 & 18.1 \\
\hline Suma & 9.9 & 16.2 & 3 & 2 & 4 & 4 & 3 & 2 & 2 & 5 \\
\hline
\end{tabular}

TABLA No 3 Valores horarios para marzo en Chinchiná (promedio de 6 años)

\begin{tabular}{||l|c|c|c|c|c|c|c|c|c|c|c|c|c|c|c|c|c|c|c|c|c|c|c|c|}
\hline \multicolumn{1}{|c|}{ HORAS } & 1 & 2 & 3 & 4 & 5 & 6 & 7 & 8 & 9 & 10 & 11 & 12 & 13 & 14 & 15 & 16 & 17 & 18 & 19 & 20 & 21 & 22 & 23 & 24 \\
\hline Cantidad (m./m.) & 15 & 23 & 23 & 19 & 15 & 9 & 8 & 6 & 3 & 1 & 8 & 5 & 12 & 4 & 7 & 3 & 8 & 14 & 7 & 7 & 13 & 14 & 4 & 8 \\
\hline Frecuencia & 4 & 6 & 6 & 6 & 6 & 5 & 3 & 2 & 2 & 1 & 1 & 1 & 1 & 3 & 2 & 4 & 3 & 3 & 4 & 4 & 3 & 3 & 3 & 4 \\
\hline Duración (Horas) & 4 & 4 & 5 & 5 & 6 & 4 & 3 & 2 & 2 & 1 & 1 & 1 & 1 & 2 & 2 & 2 & 3 & 2 & 3 & 3 & 3 & 2 & 3 & 3 \\
\hline \hline
\end{tabular}

En la tabla siguiente (tabla 4) se aclara la distribución porcentual de los elementos pluviométricos básicos en la zona húmeda, los cuales quedan enmascarados al tomar en cuenta solo las sumas mensuales como trans. curso del tiempo.

TABLA No 4 Distribución porcentual (\%) anual de cantidad, trecuencia y duración horaria:

\begin{tabular}{|c|c|c|c|c|c|c|c|c|c|c|c|c|}
\hline & Enero & Feb. & Marzo & Abril & Mayo & Junio & Julio & Agosto & Sepbre. & Ocbre. & Nov. & Dic. \\
\hline \multicolumn{13}{|c|}{ ESTACION QUIBDO } \\
\hline Cantidad $(\%)$ & 8.4 & 7.4 & 4.7 & 7.1 & 6.8 & 11.3 & 13.2 & 12.3 & 7.3 & 8.4 & 6.5 & 8.4 \\
\hline Frecuencia & 9.6 & 5.9 & 4.7 & 7.8 & 13.4 & 10.1 & 8.7 & 7.9 & 8.2 & 8.9 & 6.4 & 8.2 \\
\hline Duración & 10.0 & 5.6 & 4.8 & 6.5 & 10.6 & 9.9 & 9.3 & 9.1 & 8.9 & 9.7 & 7.2 & 8.5 \\
\hline \multicolumn{13}{|c|}{ BAJO CALIMA } \\
\hline Cantidad \% & 6.0 & 5.1 & 6.3 & 6.5 & 9.1 & 8.3 & 9.4 & 9.1 & 8.7 & 11.4 & 9.3 & 11.2 \\
\hline Frecuencia & 7.6 & 5.3 & 6.0 & 6.3 & 9.5 & 7.9 & 10.4 & 9.7 & 9.1 & 10.5 & 9.2 & 8.5 \\
\hline Duración & 6.3 & 4.8 & 5.3 & 5.7 & 10.4 & 10.3 & 9.0 & 10.4 & 9.6 & 10.1 & 8.3 & 9.8 \\
\hline \multicolumn{13}{|l|}{ RESTREPO } \\
\hline Cantidad $\%$ & 6.7 & 5.2 & 5.1 & 12.8 & 8.8 & 5.8 & 5.3 & 3.2 & 10.7 & 16.2 & 10.5 & 8.7 \\
\hline Frecuencia & 7.2 & 5.8 & 6.4 & 9.4 & 9.8 & 7.4 & 6.9 & 5.5 & 7.1 & 13.8 & 11.4 & 9.2 \\
\hline Duración & 6.6 & 5.7 & 6.0 & 10.8 & 8.8 & 7.3 & 6.5 & 4.8 & 6.8 & 14.0 & 12.7 & 10.1 \\
\hline
\end{tabular}

Las cantidades excepcionales de julio y agosto en Quibdó, de alrededor de $1000 \mathrm{~mm}$. mensuales, mostrarían que en esta época la distribución de la cantidad alcanza su máxima; pero esto no es así si se tiene en cuenta que la frecuencia y la duración de las precipitaciones disminuye notablemente. El ciclo diario de la intensidad muestra un aumento fuerte (máxima) entre las 17-19 horas, mientras que la cantidad aumenta entre las 20 y las 22 horas. El ciclo diario de todos los factores es tan marcado que no deja duda sobre la acción primor- dial de las circulaciones locales. En el Bajo Calima también se manifiesta un máximo secundario de la cantidad pluvial, en el mes de agosto, acompañado por valores altos de frecuencia y duración, pero la intensidad disminuye un poco. La máxima intensidad se registra a las 15-17 horas, lo cual se debe atribuir a la acción de la circulación local, tomando en cuenta el valor alto del brillo solar en este mes. En Restrepo la intensidad máxima se observa durante el mes de septiembre, cuando la duración del brillo solar es un poco reducida. La máxima in- 


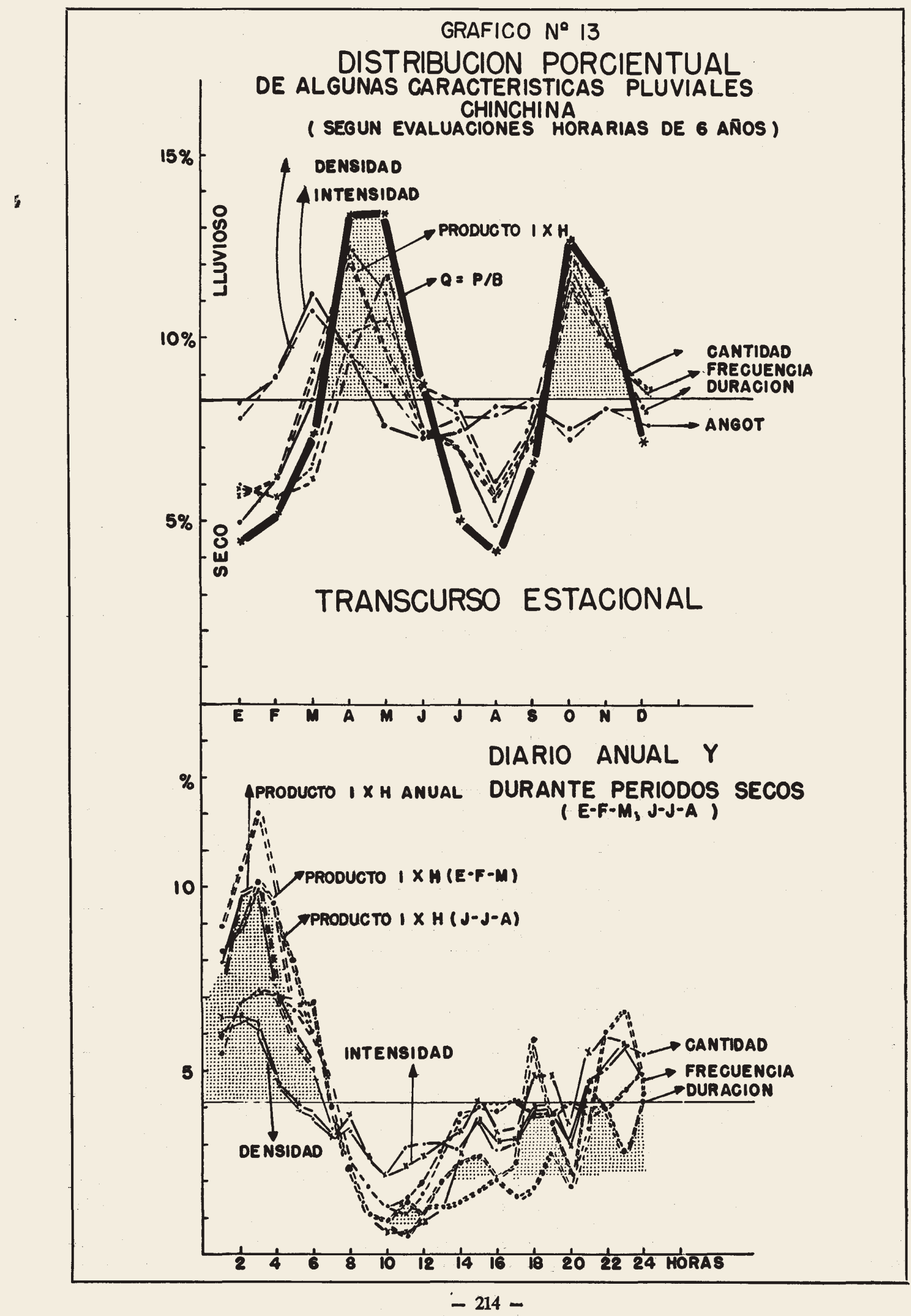




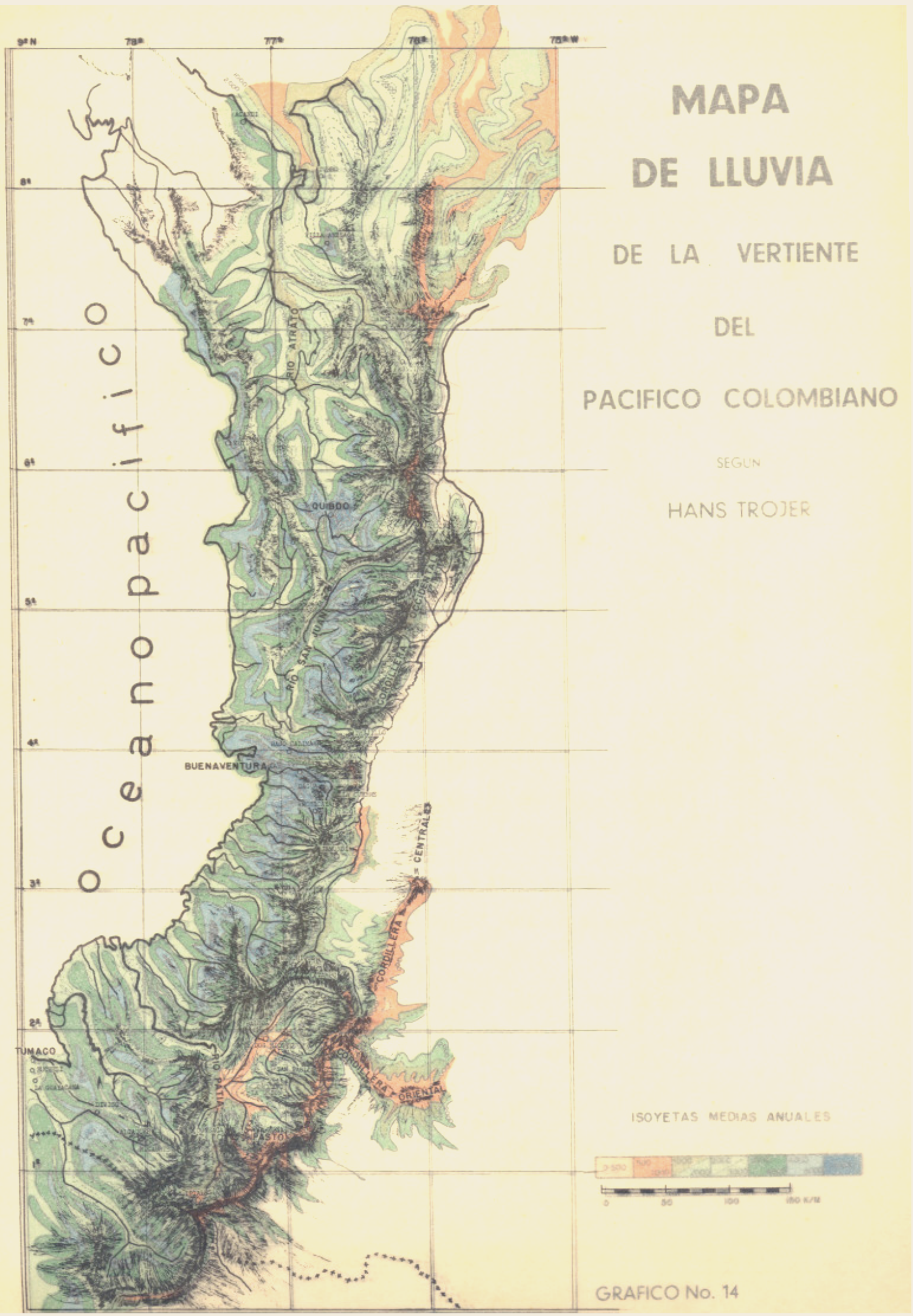


tensidad pluvial diaria se presenta entre las 13 y las 15 horas, lo cual también está de acuerdo con el desarrollo diurno de la circulación local para esta estación. En consecuencia, por un análisis detallado se obtiene una mejor interpretación de la distribución pluvial y la explicación de sus grandes diferencias a corta distancia. Además, esta interpretación global constituye una ayuda útil para el cotejo de los datos pluviométricos, los cuales a veces se ponen precipitadamente en duda por algunas pocas irregularidades encontradas.
Si se ex plea la distribución porcentual para el estudio del transcurso de los elementos, eliminando hasta el máximo la influencia permanente de la topografía, pues la distribución geográfica de los valores anuales hace resaltar en primer lugar el efecto topográfico, apesar que el transcurso predominante del macrotiempo no se puede separar en este caso, es necesario proceder a una división por zonas en las cuales varíe poco el macrotiempo correspondiente, lo cual conduce a la agrupación siguiente (tabla 5).

TABLA No 5 Precipitación pluvial anual en diferentes zonas:

\begin{tabular}{|c|c|c|c|}
\hline LUGAR & ALTURA APROXIMADA & LLUVIA & ANUAL \\
\hline \multicolumn{4}{|l|}{ ZONA NORTE } \\
\hline Turbo & 2 Mts. & 1.952 & m.m. \\
\hline Villa Arteaga & 170 & 5.055 & $"$ \\
\hline Dabeiba & 1.350 & 1.564 & $"$ \\
\hline Mutis & 5 & 5.547 & $"$ \\
\hline Quibdó & $40 "$ & 9.676 & $"$ \\
\hline \multicolumn{4}{|c|}{ ZONA CENTRAL } \\
\hline Buenaventura & 8 Mts. & 9.676 & m.m. \\
\hline Bajo Calima & 40 & 7.907 & $"$ \\
\hline Dagua & 1.000 & 5.765 & $"$ \\
\hline Anchicayá & 1.200 & 5.091 & $\overline{\prime \prime}$ \\
\hline Calima & $1.500 \cdots$ & 962 & $"$ \\
\hline La Cumbre & 1.580 & 1.274 & $\pi$ \\
\hline Restrepo & 1.670 & 1.233 & $"$ \\
\hline \multicolumn{4}{|l|}{ ZONA SUR } \\
\hline Tumaco & 3 Mts. & 2.887 & m.m. \\
\hline Buchelli & $30 "$ & 2.330 & $"$ \\
\hline La Guayana & 380 & 4.855 & $"$ \\
\hline Diviso & 800 & 4.801 & $"$ \\
\hline Altaquer & 1.070 & 3.774 & $"$ \\
\hline Ricaurte & 1.550 & 3.474 & $"$ \\
\hline Pasto & 2.590 & 984 & $"$ \\
\hline Ipiales & 2.890 & 788 & $"$ \\
\hline
\end{tabular}

La distribución de la lluvia muestra inequívocamente el régimen diferente de las zonas norte, central y sur; además, la influencia de la topografía se observa en cualquiera de las zonas anteriores, en la relación de la cantidad pluvial anual con la altura. En todos los casos la precipitación es menos en las cimas de las montañas (Calima-Madroñal 962 mm. $1.500 \mathrm{mts}$; Restrepo 1.233 mm., 1670 mts.; Pasto 885 mm. $2.590 \mathrm{mts}$.); en los fondos de los valles y en la costa (Córdoba $4.865 \mathrm{~mm}$., 50 mts.; Dos Ríos-Patía 698 mm., 600 mts., Tumaco 2.887 $\mathrm{mm} .3 \mathrm{mts}$.) que en las laderas donde actúan ambas circulaciones locales, la diurna y la nocturna. Las precipitaciones son diurnas en las partes altas, y preferiblemente nocturnas en las partes bajas.

Por configuraciones especiales del relieve se explican algunos casos de diferencias pluviales notorias a corta distancia (5 a $10 \mathrm{~km}$.) como en Quibdó: Ciudad 8.449 $\mathrm{mm}$. Aeropuerto $7.323 \mathrm{~mm}$. y el Aguillón $9.676 \mathrm{~mm}$.

En este sentido vale indicar que, observando varios registros pluviales, se encuentran formas típicas que se repiten en la misma estación o en diferentes estaciones, y que permite suponer también causas semejantes a las que las originaron. La deducción inversa, el conocimiento de las condiciones atmosféricas completas y su relación con la configuración topográfica, da las características del tiempo y de sus manifestaciones pluviales (forma de iniciación, distribución de las intensidades, duración aproximada); debido al desarrollo de estos procesos y a su obligatoria inversión, se facilita la localización más precisa de su radio de acción y el rumbo del desplazamiento.

Con base en las consideraciones anteriores sobre la actividad y el engranaje de los diferentes procesos en la atmósfera dinámica queda pendiente para la ampliación de interpretaciones generalizadas el concepto general de que a acciones semejantes resultan reacciones semejantes. Estas bases permiten realizar una interpretación preliminar, en escala conveniente, de la distribución geográfica de la precipitación pluvial (mapa de lluvia, (Gráf. 14) de la vertiente del Pacífico de Colombia, a pesar de que las observaciones disponibles son relativamente pocas (unas 80 ). También la discontinuidad de la observación durante diferentes años presenta sus 
dificultades, pero más bien cuando se trate de elaborar mapas mensuales o más detallados a escala mayor.

En situaciones semejantes de altura y de configuración topográfica se nota un aumento de las cantidades pluviales anuales como también del número de días lluviosos desde los extremos norte y sur hacia la zona central $\left(3^{\circ}\right.$ a $5^{\circ}$ de latitud $N$ ) puesto que esta zona permanece aun influenciada por las vagüadas de las perturbaciones cuando estas se hallan aun en sus posiciones extremas, Norte o Sur. Por lo tanto, la actividad pluvial en la zona central es excepcional, pues el estancamiento de las corrientes generales, la convergencia por la topografía y el buen desarrollo de las circulaciones locales, refuerzan gradualmente la intensidad de la lluvia de acuerdo con las perturbaciones correlacionadas con la ITC. Estas condiciones se pueden suponer óptimas en el segundo tercio de altura relativa de las laderas (entre 50 a 1.000 mts.). Según los datos pluviométricos, se observan más de $5.000 \mathrm{~mm}$. anuales en las siguientes estaciones: Villa Arteaga $5.055 \mathrm{~mm}$. alt., $170 \mathrm{mts}$; Quibdó 8.449 mm. 40 mts.; Itsmina 9.333 mm. 60 mts.; Andagoya $6.784 \mathrm{~mm} .40 \mathrm{mts}$; Bajo Calima $6.921 \mathrm{~mm} .40 \mathrm{mts}$; Dagua 5.765 mm. 800 mts.; Anchicayá 5.091 mm. 1.000 mts.; El Diviso $4.801 \mathrm{~mm} .800 \mathrm{mts}$.

Como el efecto de estancamiento es débil en las partes bajas y el contenido de agua en la atmósfera es mayor, y en contraste, en las partes altas es menor el contenido de agua pero el efecto de estancamiento es mayor, las cantidades de lluvia en estos niveles son casi iguales. Sin embargo, al estudiar las características completas (5) de su régimen (duración, intensidad, horas de iniciación, horas de mayor frecuencia, etc.) podrán diferenciarse zonas con las mismas características, fácilmente relacionables como manifestaciones conjuntas del mismo tiempo reinante.

\section{DEFINICION CLIMATOLOGICA DE LAS ZONAS HUMEDAS}

Los datos de cantidad, distribución y número de días lluviosos no siempre son tan indicativos de las características climatológicas de una región, como su relación conjunta y más detallada según las manifestaciones completas del tiempo; es decir, por medio de una verdadera interpretación dinámica.

Esta interpretación promete, tal vez, una mejor definición climática del concepto de las zonas húmedas tropicales, que los cuocientes o definiciones acostumbrados, los cuales en todo caso tienen que considerar también el transcurso meteorológico, indicando la presencia permanente o temporal de períodos húmedos. Así, se pueden considerar como períodos super-húmedos solo las épocas en las cuales predomina notoriamente el tiempo lluvioso con todas sus manifestaciones características. Para presentar el grado del tiempo lluvioso se puede aplicar un cuociente ya experimentado (11) para relaciones entre la acción climática y la reacción de la vegetación, según la combinación de los valores de precipitación y de la duración del brillo solar $(\mathrm{P} / \mathrm{B})$.

Este cuociente que puede aplicarse a sumas anuales, mensuales o a cualquier período del mismo carácter del tiempo, indica, por lo tanto, el estado promedio y también el transcurso de los diversos períodos estacionales. Se correlacionan en esta forma dos elementos muy im- portantes con transcurso propio muy marcado, y sus valores reflejan muy bien la influencia de las variaciones locales. La ventaja esencial del cuociente $\mathrm{P} / \mathrm{B}$ está en su mejor expresión de la efectividad de la precipitación de acuerdo con el efecto de la radiación (brillo solar): un mes con alta cantidad pluvial, pero al mismo tiempo con una duración media del brillo solar, da un cuociente bajo y es en su efectividad respecto al suelo y a la planta muy diferente a un mes con igual cantidad pluvial y poco brillo solar (cuociente alto). Cuanto más alto es el cuociente, más húmedo es el período. Como ejemplo de una diferenciación por el cuociente se pueden mostrar los siguientes datos: Galerazamba - Bolívar - Costa Atlántica, representa un cuociente de 0.40; para Chinchiná, Caldas en la zona cafetera, el cuociente es de 1.43; en el Bajo Calima - Valle - zona húmeda del Pacífi$\mathrm{co}$, es de 5.83. Si el cuociente es durante todo el año muy alto (mayor de 2.5) es decir, cuando la duración del brillo solar es solo $1 / 4$ del valor de la cantidad pluvial, indica que en cualquier zona la reducida insolación no alcanza a secar el suelo y el aire, presentando tales regiones las características especiales de tierras húmedas. Esta definición preliminar incluye también las zonas de alturas elevadas donde la precipitación es menor pero también la insolación es muy pequeña debido a la frecuente nubosidad que obliga a determinar estas regiones también como húmedas, como lo demuestra la vegetación (zonas altas). En cuanto a las zonas húmedas causadas por inundaciones temporales donde el aire adyacente a la zona inundada acumula bastante vapor acuoso que se traduce en frecuente nubosidad local, y reduce notoriamente la duración del brillo solar, por lo cual se aumenta de esta manera el cuociente, es necesario realizar observaciones especiales que permitan averiguar hasta qué límite la expresión del cuociente las caracteriza. En este sentido, vale la pena comparar las diferentes clasificaciones conocidas y el cuociente $\mathrm{P} / \mathrm{B}$ con la clasificación ecológica.

\section{$R E S U M E N$}

Partiendo de los conocimientos generales de la $\mathrm{Me-}$ teorología y de la descripción de los fenómenos básicos del d-Trollo del tiempo reinante en el trópico, se puede demostrar que las condiciones meteorológicas en las zonas tropicales húmedas, son apenas una variación especial de los fenómenos comunes debido a excepciones en la combinación de los factores básicos que determinan el tiempo en cualquier momento y para cualquier lugar.

Estos factores son: A) El macrotiempo, la situación momentánea de los centros de presión atmosférica (posión del sistema intertropical de circulación y de la ITC) que determinan la dirección de la translación horizontal, la estructura de las masas de aire y la evolución de los efectos hidrodinámicos.

A.- a) Fenómenos locales producidos por la topografía según el macrotiempo.

B) Sistemas locales de circulación recíproca debidos a diferencias térmicas locales.

Estos factores generales son excepcionales en las zonas humedas tropicales y las propiedades tipicas de las masas de aire (inestabilidad vertical) a lo cual se adicionan los fuertes efectos de estancamiento y también las condiciones favorables para la evolución de las circulaciones locales por la gran inestabilidad de las masas, determi- 
nan las características propias de estas regiones. Las masas de aire ecuatorial del Pacífico presentan tales circunstancias y según la distribución bárica son movilizadas por la corriente general hacia los subtrópicos, donde convergen a lo largo de la ITC con masas subtropicales más frescas pero menos húmedas debido a su translado de norte a sur.

En la linea de convergencia se forman centros parciales de baja presión, los cuales dan origen al tiempo ciclonal con fuerte reacción en las manifestaciones meteorológicas, debido a que las pequeñas diferencias térmicas y a la alta humedad del aire producen las condensación de enormes cantidades de vapor acuoso.

Las condiciones especiales de la topografía que favorecen los efectos de estancamiento y del desarrollo de circulaciones locales, aumenta la pluviosidad y prolongan las lluvias a días de transición en lugares determinados, cubriendo franjas de unos 5 a $10 \mathrm{~km}$. tal como se localizan en el mapa de lluvia. Durante la influencia de las zonas anticiclonales tampoco se presentan precipitaciones en estas regiones como confirman las observaciones de Tumaco donde se presenta en periodo seco de dos semanas (1): sólo se observa la formación de nubes de ciclo diario sobre los focos regionales principales. Asi se explican las diferencias locales de la tántidad pluvial a pocos kilómetros, las cuales sobresalen en determinados lugares debido a la permanente y más frecuente acción - de las relaciones entre la topografía y el desarrollo del tiempo.

El transcurso del tiempo reinante (tiempos lluviosos y secos) están inequivocadamente correlacionado con el translado cíclico del sistema de circulación intertropical a través del año, en su movimiento casi sincronizado con el del sol.

La ITC no se presenta como una línea o franja fija, como se supone en el sentido climatológico según. el concepto de los Alisios, sino que está en permanente movilización por el dinamismo de los procesos atmosféricos y se observa una continua destrucción y rejuvenecimiento de sus perturbaciones atmosféricas en la misma forma como ocurren realmente los cambios diarios del tiempo, aún durante los periodos de mayor actividad pluvial.

A principio del año la época lluviosa se presenta en el sur del territorio Costero del Pacífico y el carácter ciclonal del tiempo avanza paulatinamente hacia el norte, alcanzando en promedio la latitud de Buenaventura hacia finales de Abril y principios de Mayo, para manifestarse plenamente en la parte norte de la zona hacia mediados del año. En septiembre comienza su regreso hacia el sur, haciéndose más agudo hacia octubre y noviembre. De año en año pueden observarse modificaciones en la duración e intensidad de las épocas ciclonales $y$ anticiclonales, según la posición variada de todo sistema de circulación tropical. Si durante un año, o parte de él, este sistema se encuentra relativamente en posición septentrional, este lapso se presenta menos lluvioso debido a la influencia que ejerce la zona de alta presión de cinturón meridional avanzado con sus cuñas sobre ol territorio colombiano.

Los valores climatológicos correspondientes a las zonas húmedas del Pacífico son: Temperaturas medias anuales de 25 a $27^{\circ}$ en la costa, y algo más altas (27-29\%) en los bajos de los valles debido al aumento de insola- ción en estas zonas. En general la disminución de la temperatura con la altura desde 0.5 a $0.6^{\circ} \mathrm{C} / 100 \mathrm{~m}$. aproximadamente. La oscilación diaria es muy pequeña y aún mayor en los valles que en las laderas ( 6 a $8^{\circ} \mathrm{C}$ y 4 a $6^{\circ} \mathrm{C}$ ) respectivamente durante buen tiempo): humedad relativa media en toda la zona oscila entre 80 y $90 \%$, la tensión del vapor se eleva de 22 a $24 \mathrm{~mm} . / \mathrm{Hg}$, lo cual hace sufrir al hombre por el ambiente sofocante: también la duración del brillo solar se reduce a máximos de 6 a 8 horas (valles) como consecuencia de la frecuente formación de nubes de ciclo diurno, pero sin embargo se presentan tardes despejadas con 5 horas de brillo solar después de una mañana lluviosa.

La distribución pluvial (ver mapa de lluvia) muestra el régimen diferente de las zonas Norte, Central y Sur: además de la influencia de la topografía, se observa en cualquiera de las zonas anteriores la relación de la cantidad pluvial anual con la altura. En todos los casos la precipitación es menor en las cimas de las montañas, en los fondos de los valles y en la costa, que en las laderas donde actúan ambas circulaciones locales, la diurna y la nocturna. Para el estudio de las características completas del régimen pluvial se requieren datos sobre duración, intensidad, horas de iniciación y horas de mayor frecuencia para diferenciar las zonas con características semejantes, fácilmente relacionables como manifestaciones conjuntas de todos los elementos del mismo tiempo reinante.

Vale la pena intentar una clasificación de las zonas húmedas por medio del cuociente $P / B$ (precipitación, brillo solar) y su relación con la vegetación, pues así se combinan dos elementos meteorológicos importantes de los cuales cada uno representa en cierto grado el tiempo reinante, como se nota por ejemplo, con los valores de Galerazamba (Costa Atlántica) que tiene un cuociente de 0.40: la zona Cafetera (Chinchiná) con 1.43 y el bajo Calima en la zona húmeda del Pacífico con 5.83, de lo cual se deduce que los meses con un cuociente mayor de 2.5 son húmedos. También, se puede esperar que debido a la reducción de la duración del brillo solar a causa del aumento de la nubosidad local, los valores del cuociente reflejarán las características de las zonas frecuentemente inundables.

\section{$S U M M A R Y$}

According to the general knowledge on meteorology and the descriptions of the basic phenomena on the development of the weather types in the tropics, the meteorological conditions in humid tropical zones are only a special variation of the ordinary phenomena. These are due to exceptions of the combination of the basic factors which determine the weather in any time and at any place.

These factors are: A) The general weather situation, the momentary situation of the centre of the atmospheric pressure (position of the intertropical system of circulation and the ITC) which determine the direction of the horizontal shifting as well as the structure of the respective air masses and the development of the hydrodynamical effects.

A.-a) The local phenomena produced by the topography according to the general weather.

$B)$ The local circulation systems due to local thermic differences with daily periodical changes. 
These factors are exceptional in the humid tropical zones. The typical characteristics of air masses (vertical instability) to which are to be added strong stammings effects and favorable conditions for the development of the local circulations in consequence of strong instability of the air masses, determine the characteristics of these regions. The equatorial air masses from the Pacific possess such conditions and are shifted with the general current to the subtropics, in accordance with the baric distribution where they meet with subtropical air masses along the ITC, which are fresh but less humid as the result of its shifting direction from north to south.

Partial centres of low pressure form themselves in the convergence line and originate the ciclonal weather with strong reactions on the meteorological manifestations. This occurs in spite of the little horizontal differences of temperature but with a high moisture content the vertical movements cause a condensation and precipitation of enormous quantities of water.

These special topographic conditions facilitate the stamming effects and therefore the evolution of local circulations. Both increase the quantity of precipitations and multiply the frequence in determinate places $d u$ ring days of transitional weather and cover zones of 5 to $10 \mathrm{~km}$. as may be seen in the rainfall map. During the influence of anticiclonal weather also in these regions occur dry periods, as confermed from data from Tumaco registering periods of even two weeks without precipitations (1). However, cloud formations are observed above special regional focuses having a determinate daily cicle. This explains the very local differences of the rainfall distribution within a few kilometres only, which is being accentuated in the yearly rain sums of determinate places in consequence of permanent and frequent influences of the topography on the sequences of the weather.

The sequence of weather (rain weather and dry weather season) is doubtless correlated with the annual shifting cycle of the intertropical circulation system. These movements are almost synchronized with those of the sun position.

The Intertropical Convergence Line $-I T C-$ is not fixed in form of a line or a belt as being supposed in the climatological conception of the trade-wind-belts, but is permanently moving by reason of the dynamic of the processes in the atmosphere. This fact is perceptible through the continous destructions and renovals occuring in the atmospheric perturbations just as they occur during daily weather changes also during the strongest rain periods.

The rainy season may be observed at the beginning of the year in the southern Pacific coast and the cyclonal weather advances by degrees towards north reaching Buenaventura latitude at about the end of April or at the beginning of May. In the middle of the year it reaches a complete development in the northern part of this zone. During September the rainy season begins to return towards south, being generally accentuated during Octuber and November. From year to year modifications are being observed in respect to the duration and intensity of the cyclonal and anticyclonal periods, according to the changed position of the tropical circulation system. When during a year or part of it the ITC with all the intertropical system is established in his relatively northern position, then this period is less rainy because the colombian territory is under the influence of the zone of the southern high pressure belt.

The climatological values of the humid zones of the Pacific coast are the following: annual mean values of temperature 25 to $27^{\circ} \mathrm{C}$ in the coast and a little higher, $27-29^{\circ} \mathrm{C}$, in the low land of the valleys, due to the increase of insolation in this regions. Generally the decrease of temperature with the altitude is approximately 0,5 to $0,6^{\circ} \mathrm{C} / 100 \mathrm{~m}$. The daily oscillation is very small but still a little more accentuated in the valleys than on the slopes (during fine weather $6-8^{\circ} \mathrm{C}$ and $4-6^{\circ} \mathrm{C}$ respectively). The mean weather values of relative humidity in the entire zone is between 80 and $90 \%$; the vapor tension reach in the mean values fron 22 to $24 \mathrm{~mm}$. $\mathrm{Hg}$. and these weather conditions produce in human beings a feeling of sultry oppression. The duration of sunshine decrease on a maximum of 6 to 8 hours (in the valleys) due to the frequent formation of clouds with a diurnal cicle. But after mornings with rainfalls, followed by sudden clearings at noon time, cloudless afternoons with up to 5 hours of sunshine occur.

The distribution of precipitations (see rain map) shows the difference between the north, central and south part of the region. There may also be observed the topographical influence in this relation on the annual pluvial quantity with the altitude in each region. In any case the precipitation is always lower at the summit of the mountains, in the valleys and on the shores than on the slopes where the diurnal and the nocturnal system of local circulation is active. For a complete study of the characteristics of the precipitation would be therefore necesary data on duration, intensity, hours of initiation, and frequency in the different zones having the same characteristics. These data would make it possible to relate the common manifestations in all the meteorological elements during the same general weather situations.

It would be interesting to check a classification of the humid zones according to the quotient $P / B$ (precipitation-Sunshine duration) and its relation to the vegetation. This quotient uses two important meteorological elements where each represent to certain degrees the predominant weather. This can be recognized in the following examples: Galerazamba (Atlantic Coast) with a quotient 0,40; the Coffee Zone (Chinchina Caldas) 1,43 and Bajo Calima in the humid zone of the Pacific 5,83. In consequence humid months have a quotient higher than 2,5. Besides it could be possible that the decrease of sunshine duration due to local cloud formations above zones of frequent inundations are also shown through the values of this quotient.

\section{BIBLIOGRAFIA}

(1) CASTRO TORRIJOS R.: Chocó - Colombia. Mimeografiado Contraloría General del Chocó. (Simposio de las tierras Húmedas Tropicales; Quibdó 1958).

(2) HANN J.: Handbuch der Klimatologie. I. Teil Klima der Tropenzone. Stutgart; Verlag von ENGELHORN, 1910.

(3) KÖPPEN W. GEIGER R.: Handbuch der Klimatologie. Bd. II. Teil: Klimakunde Von Südamerika. Berlín, Verlag Gebrüder Borntraeger 1930. 
(4) PATIÑO V.: Presentación del Calima. Publicaciones de Secretaría de Agricultura del Valle; Cali 1946.

(5) PEREZ ARBELAEZ E.: Recursos naturales en Colombia: Capítulo Clima, Cita H. Trojer, Bogotá, Banco de la República 1954.

(6) TROJER H.: Ein Beitrag zu einer dinamischen Klimatologie, Publicaciones del Observatorio del Ebro, Tortosa, España 1949.

(7) TROJER H.: El tiempo reinante en Colombia, Boletín Técnico Federación Nal. de Cafeteros, Vol. II-13, 1954.

(8) TROJER H.: El ambiente climatológico y el cultivo del café en Colombia, Bol. Inf. Centro Nal. de Inv. de Café, Vol. V, N 57, 1954.

(9) TROJER H.: Nuevo rumbo en la Climatología Tropical y su importancia en las Ciencias del suelo. Bol. Inf. Centro Nal. de Inv. de Café, Vol. VI.

(10) TROJER H.: Distribución horizontal de luminosidad en un cafetal y en almácigos.
Boletín Informativo Vol. IV No 45, Centro Nal. de Investigaciones de Café, 1953.

(11) TROJER H.: Investigación Agroclimatológica para el cultivo del Café en Colombia. Bol. Inf. № 75, 1956.

(12) TROJER H.: Informe Técnico del Plan Lilienthal: Parte de Meteorología, Archivo Corporación Autónoma del Valle del Cauca, 1956.

(13) WEST ROBER'T C.: The Pacific Lowlands of Colombia. Lousina State University Studies No 8, 1957.

(14) ANUARIOS: Contraloría de la República, Anuario general de estadística 1949-1950-1951.

Ministerio de Agricultura, Anuarios Meteorológi$\cos$ 1937-1954.

Instituto Nal. de Aprovechamiento de Aguas y Fomento Eléctrico y Secretaría de Agricultura del Valle, Bol. Hidrológico, 1949-1952.

Federación de Cafeteros, Anuarios Meteorológicos, 1951-1957. 